\title{
Material aspects of dynamic neck retardation
}

\author{
Z. Xue, A. Vaziri, J.W. Hutchinson* \\ School of Engineering and Applied Sciences, Harvard University, Cambridge, MA 02138, USA
}

Received 15 November 2006; accepted 18 February 2007

\begin{abstract}
Neck retardation in stretching of ductile materials is promoted by strain hardening, strain-rate hardening and inertia. Retardation is usually beneficial because necking is often the precursor to ductile failure. The interaction of material behavior and inertia in necking retardation is complicated, in part, because necking is highly nonlinear but also because the mathematical character of the response changes in a fundamental way from rate-independent necking to rate-dependent necking, whether due to material constitutive behavior or to inertia. For rate-dependent behavior, neck development requires the introduction of an imperfection, and the rate of neck growth in the early stages is closely tied to the imperfection amplitude. When inertia is important, multiple necks form. In contrast, for rate-independent materials deformed quasi-statically, single necks are preferred and they can emerge in an imperfection-free specimen as a bifurcation at a critical strain. In this paper, the interaction of material properties and inertia in determining neck retardation is unraveled using a variety of analysis methods for thin sheets and plates undergoing plane strain extension. Dimensionless parameters are identified, as are the regimes in which they play an important role.
\end{abstract}

(C) 2007 Elsevier Ltd. All rights reserved.

Keywords: Necking; Ductility; Inertia; Strain hardening; Strain-rate hardening

\section{Introduction: preview of the roles of strain and strain-rate hardening and inertia}

In this Introduction a family of visco-plastic solids will be discussed whose behavior in uniaxial tension is specified by

$$
\sigma=\sigma_{R} \varepsilon^{N}\left(\dot{\varepsilon} / \dot{\varepsilon}_{R}\right)^{m},
$$

where $\sigma$ is the true stress, $\varepsilon$ is the logarithmic strain and $\dot{\varepsilon} \equiv \mathrm{d} \varepsilon / \mathrm{d} t$ is the strain-rate with $t$ as time. Elasticity is neglected and the material is taken to be incompressible. The parameters characterizing the solid are the reference flow stress and strain-rate, $\sigma_{R}$ and $\dot{\varepsilon}_{R}$, the strain hardening exponent, $N$, and the strain-rate hardening exponent, $m$. The uniaxial relation (1) is generalized to multi-axial states in the standard manner with strain increments proportional to the stress deviator, $s_{i j}=\sigma_{i j}-\sigma_{k k} \delta_{i j} / 3$.

\footnotetext{
${ }^{*}$ Corresponding author. Tel.: + 16174952848 ; fax: + 16174960601.
}

E-mail address: hutchinson@husm.harvard.edu (J.W. Hutchinson). 


\subsection{Two-state, long wavelength approximation for visco-plastic, inertia-free stretching}

We begin by reviewing results from a simple approximate theory of neck development that captures many trends relevant to neck retardation as dependent on strain and strain-rate hardening under quasi-static stretching, i.e. stretch rates sufficiently slow such that inertia is not important. If the wavelength of the neck is sufficiently long compared to the thickness of the sheet, the sheet is everywhere in a state of approximate plane strain tension. This approximation was exploited by Marciniak and Kuczynski (1967) in their analysis of necking in sheet metal and by Hutchinson and Neale (1977) in their study of necking in materials characterized by (1). With reference to the sketch in Fig. 1, denote variables at the developing neck where the thickness of the sheet is minimum by $A$ and those in the thick, nominally uniform sections well away from the neck by $B$. Approximate plane strain tension everywhere implies $\sigma_{B} h_{B}=\sigma_{A} h_{A}$ by elementary equilibrium, where $h_{A}$ and $h_{B}$ are the current thicknesses of the sheet at the respective locations. Denote the initial thickness of the sheet at the same material sections by $h_{A}^{0}$ and $h_{B}^{0}$, and introduce the geometric imperfection amplitude as $\eta_{0}=$ $1-h_{A}^{0} / h_{B}^{0}$ with $\eta_{0}>0$. With $\varepsilon_{A}$ and $\varepsilon_{B}$ denoting the logarithmic strains at these same sections, it is elementary to show

$$
\begin{array}{ll}
\varepsilon_{B}^{N} \mathrm{e}^{-\varepsilon_{B}}=\left(1-\eta_{0}\right) \varepsilon_{A}^{N} \mathrm{e}^{-\varepsilon_{A}} & \text { for } \quad m=0 \\
\int_{0}^{\varepsilon_{B}} \varepsilon^{N / m} \mathrm{e}^{-\varepsilon / m} \mathrm{~d} \varepsilon=\left(1-\eta_{0}\right)^{1 / m} \int_{0}^{\varepsilon_{A}} \varepsilon^{N / m} \mathrm{e}^{-\varepsilon / m} \mathrm{~d} \varepsilon & \text { for } \quad m>0 .
\end{array}
$$

Note that the relation between the strains in the two sections is independent of time and, therefore, does not depend on the rate of straining. The relation is plotted in Fig. 1 for various values of the strain-rate hardening exponent with $N=0.1$ and $\eta_{0}=0.005$.

There is a fundamental difference between the response of rate-independent and rate-dependent materials. For $m=0$, the strain outside the neck reaches a limit point when $\varepsilon_{A}=N$, and, if elasticity were included, elastic loading would occur away from the neck beyond this point. By contrast, if $m>0$, the maximum strain attained outside the neck occurs asymptotically as $\varepsilon_{A} \rightarrow \infty$. Infinite ductility is assumed, and the necking strain, $\varepsilon_{\mathrm{NECK}}$, is identified with the maximum value of $\varepsilon_{B}$, corresponding to the limit point when $m=0$ and the

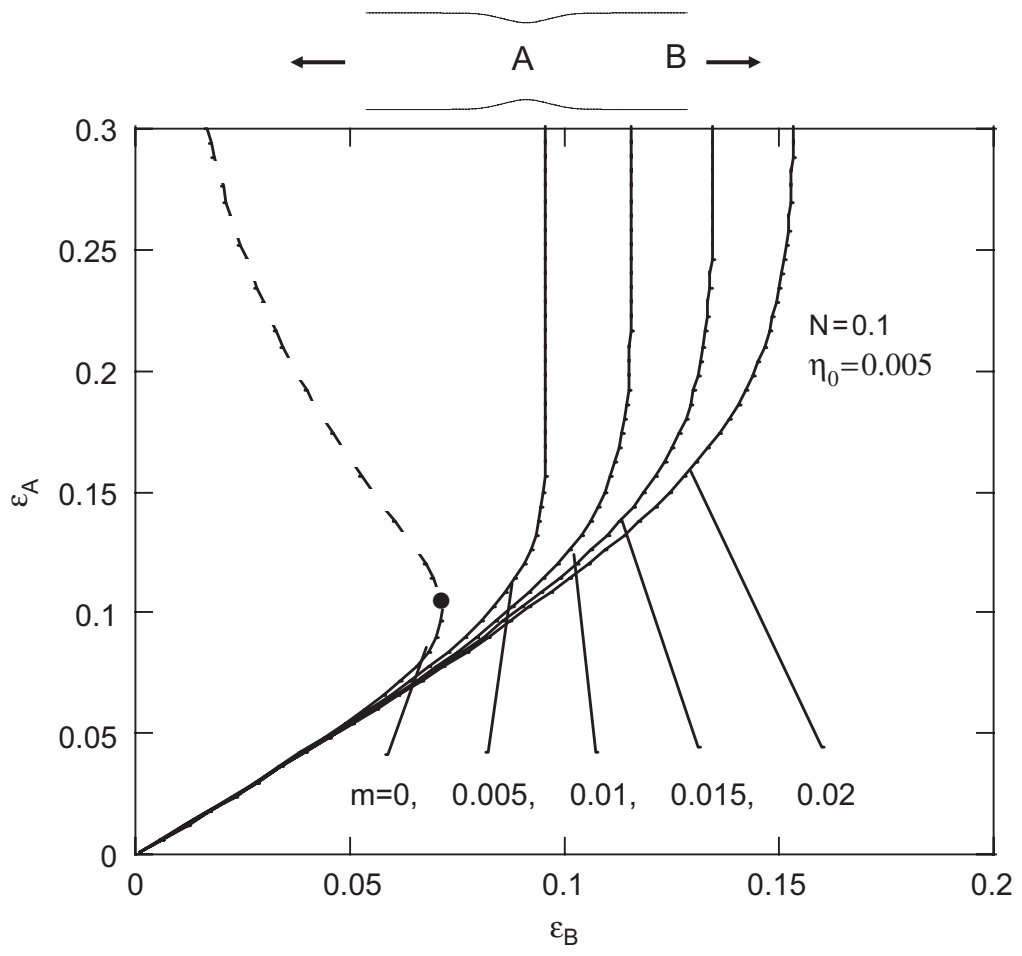

Fig. 1. Strain at the minimum section (A) as a function of the strain at the thickest section (B) for various material rate-dependencies including the rate-independent limit, $m=0$, according to the two-state model for the power law stress-strain relation (1). The results are quasi-static in the sense that inertia is neglected. 
asymptotic limit for $m>0$. The role of the initial imperfection is illustrated in Fig. 2 with $N=0.1$. A fundamental difference between the behavior of rate-independent and rate-dependent materials again emerges. In the limit when the imperfection becomes zero, $\eta_{0} \rightarrow 0$, the necking strain of the rate-independent material has the well defined limit, $\varepsilon_{\mathrm{NECK}}=N$, while it is unbounded for the rate-dependent material. Analytical asymptotic expressions derived from (2) for various functional dependencies on $N$ and $m$ for small $\eta_{0}$ are given by Hutchinson and Neale (1977).

It is evident from Figs. 1 and 2 that rate-dependence has a profound influence on neck retardation. Moreover, the retardation has strong dependence on the magnitude of the imperfection when rate-dependence is important. The relevance of rate-dependence to total elongation attainable prior to necking is highlighted by Fig. 3 where the lower plot presents experimental results for total elongation prior to necking in uniaxial tension for wide selection of ductile alloys assembled by Woodford (1969). The upper plot in Fig. 3 presents the elongation from (2), modified for uniaxial tension and approximated as $\mathrm{e}^{\varepsilon_{\mathrm{NECK}}}-1$.

\subsection{Exact perturbation results for growth of plane strain necks}

For a plate of the power-law viscous material (1) with $N=0$ and $\sigma=\sigma_{R}\left(\dot{\varepsilon} / \dot{\varepsilon}_{R}\right)^{m}$, Hutchinson et al. (1978) used perturbation theory to derive the rate of growth of a small, symmetric sinusoidal thickness undulation as depicted in Fig. 4 and specified at the current instant by $\Delta h=\frac{1}{2} \eta_{0} h \cos (2 \pi x / L)$, where $h$ is the current average thickness. The increment of growth of the thickness is given by

$$
\dot{h}=\frac{1}{2} m \eta_{0} \dot{\bar{\varepsilon}} G(m, h / L) \cos (2 \pi x / L)
$$

with $\dot{\bar{\varepsilon}}$ as the overall strain increment. The function $G(m, h / L)$ is plotted in Fig. 4 where it is seen that undulations with the longest wavelength, $L$, grow the fastest. The result for the long wave length limit can be obtained from (2) for small imperfections in the early stage of neck growth.

For rate-independent materials, Hill and Hutchinson (1975) used perturbation theory to obtain exact results for necking bifurcations from a uniform incompressible plate subject to plane strain stretching. At the instant of bifurcation, let $h$ be the thickness of the plate, $E_{\mathrm{t}}$ be the instantaneous tangent modulus relating true stress and logarithmic strain increments in plane strain according to $\dot{\sigma}=E_{\mathrm{t}} \dot{\varepsilon}$, and $\mu$ be the instantaneous modulus governing shear parallel to the plate edges. The bifurcation modes have a sinusoidal dependence, $\cos (2 \pi x / L)$,

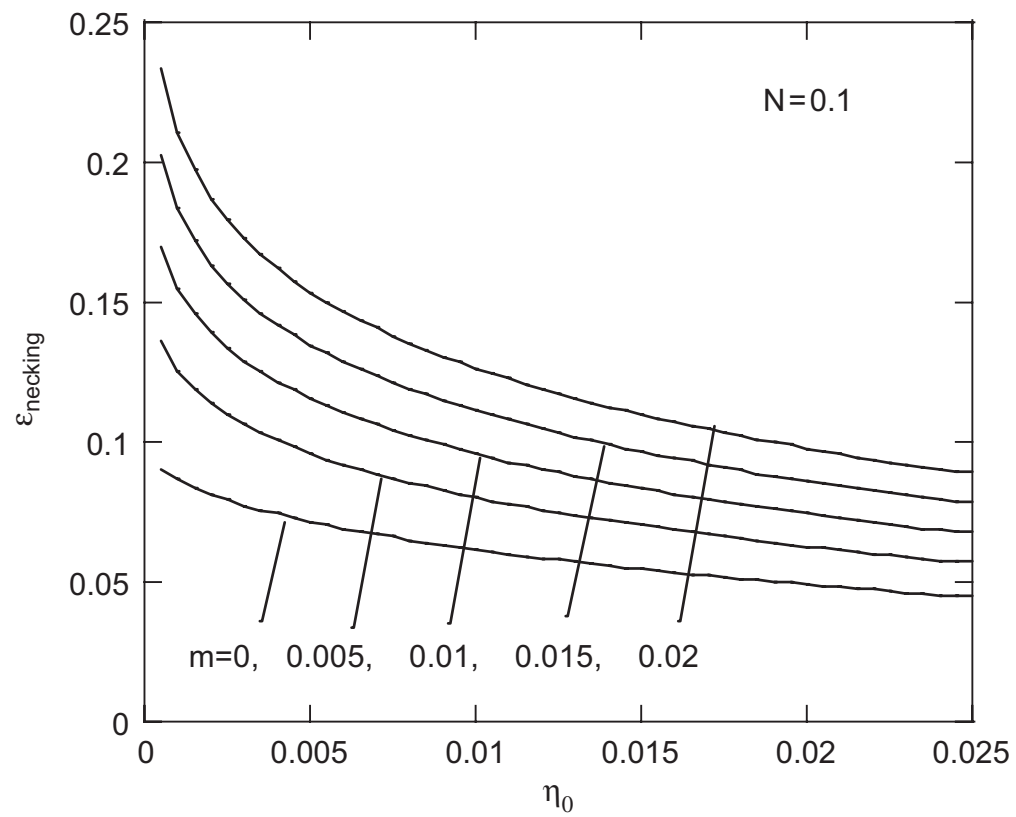

Fig. 2. Effect of initial imperfection, $\eta_{0}$, on the quasi-static necking strain according to the two-state model for the power law stress-strain relation (1). The necking strain is unbounded as $\eta_{0} \rightarrow 0$ for rate-dependent materials $(m>0)$, but $\varepsilon_{\text {necking }}=N$ as $\eta_{0} \rightarrow 0$ for the rateindependent material $(m=0)$. 

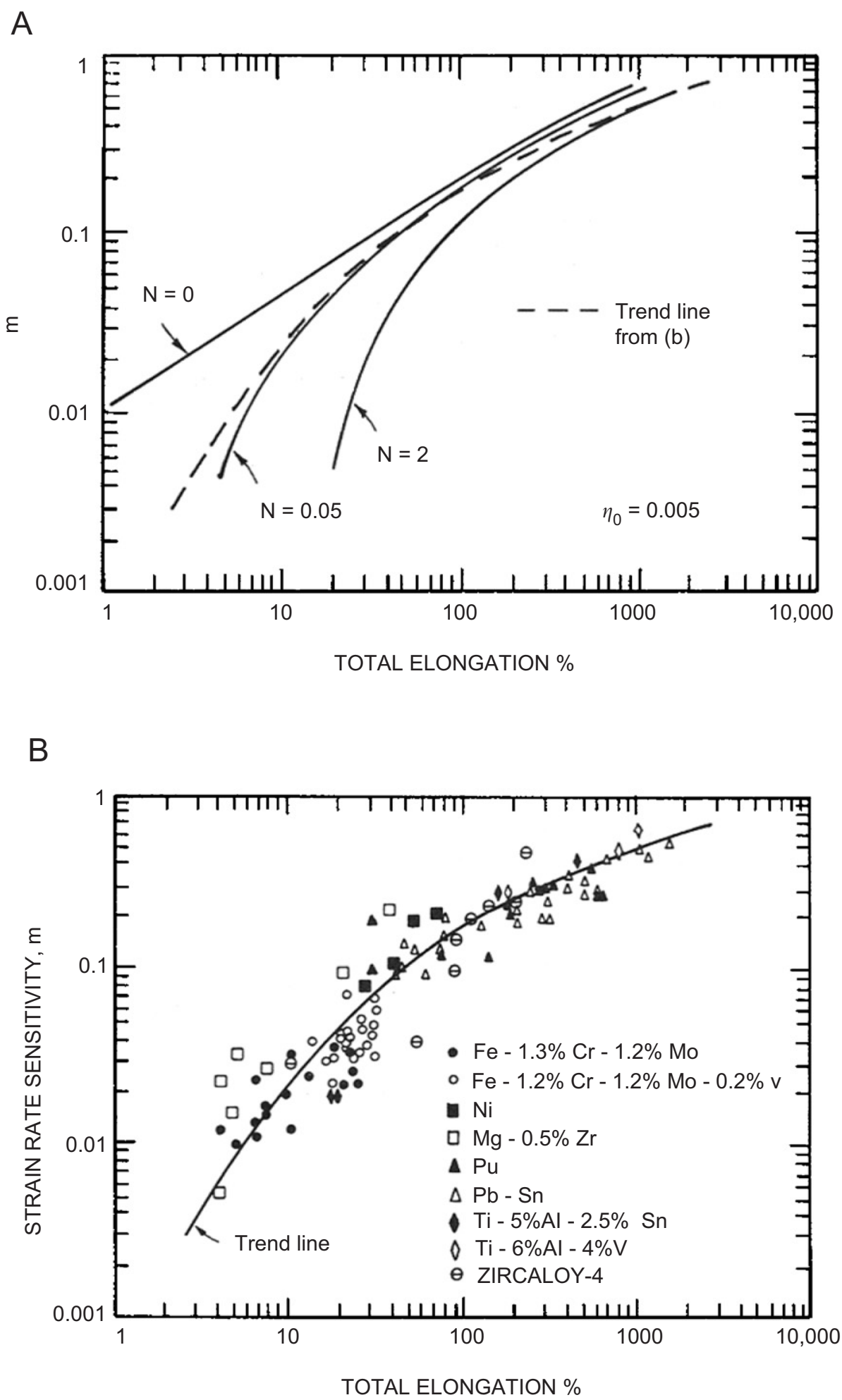

Fig. 3. Total elongation in a uniaxial tension test (change in length divided by initial length) as a function of the strain-rate hardening exponent $m$ in (1). (A) Theoretical prediction from the two-state model for three values of the strain hardening exponent. (B) Experimental data collected by Woodford (1967) for a wide assortment of rate-dependent metal alloys.

in the direction of stretching, and the functional form of the stress at bifurcation is $\sigma / E_{\mathrm{t}}=f\left(E_{\mathrm{t}} / \mu, h / L\right)$. A plot of this dependence is given in Fig. 5. The lowest bifurcation stress is always associated with the long wavelength limit, which is the classical result of Considère, $\sigma / E_{\mathrm{t}}=1$, associated with attainment of the 

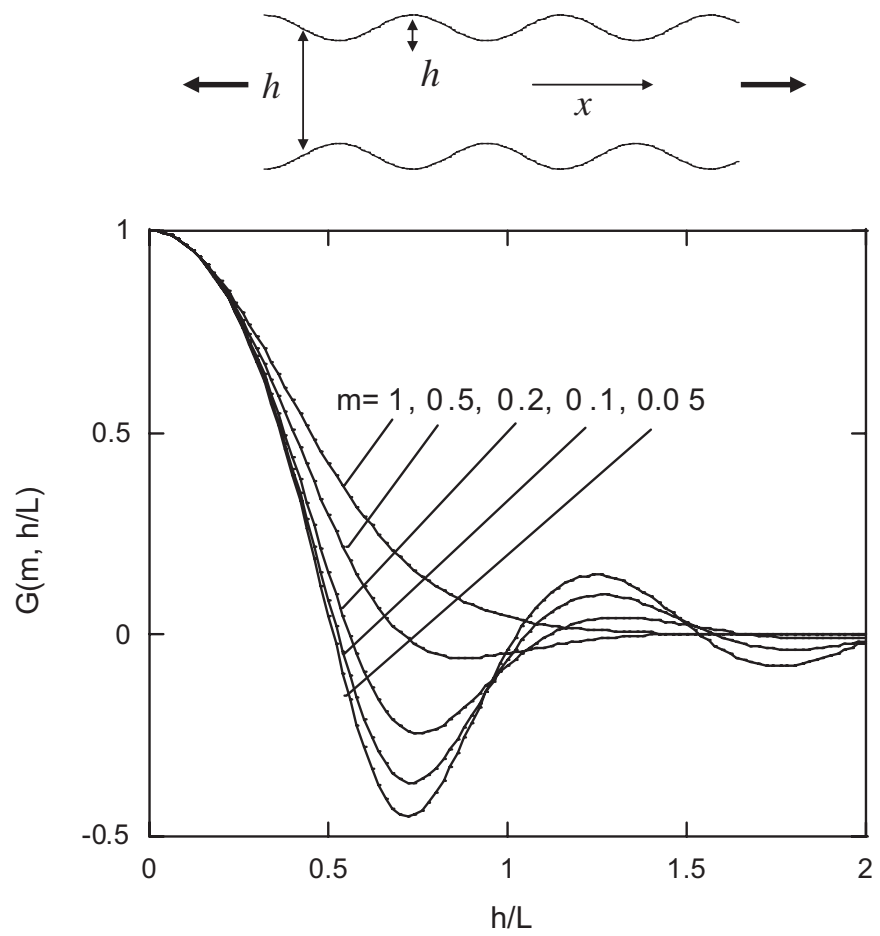

Fig. 4. Function defined in (3) determining the quasi-static growth rate of small sinusoidal undulations with wavelength $L$ for power law rate-dependent materials specified by (1) with $N=0$. The average plate thickness is $h$. Undulations with the longest wavelengths grow the fastest (Hutchinson and Neale, 1977).

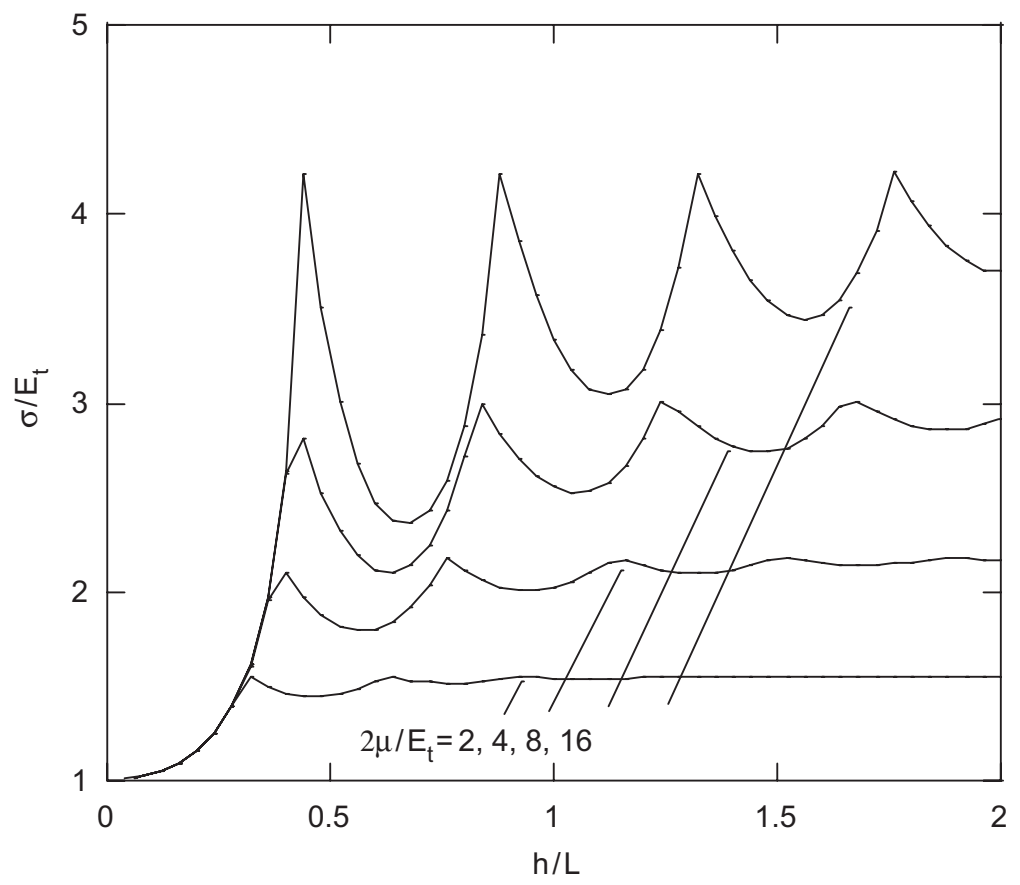

Fig. 5. Bifurcation stress at the onset of necking in a perfect plate deformed quasi-statically to the current state having tangent modulus, $E_{\mathrm{t}}$, shear modulus, $\mu$, plate thickness, $h$, and sinusoidal bifurcation mode wavelength, $L$ (Hill and Hutchinson, 1975).

maximum load. For a solid based on (1) with $m=0$ and generalized to multi-axial states by deformation theory plasticity, $2 \mu / E_{\mathrm{t}} \cong 1 / 2 N$ (Storen and Rice, 1975).

Under quasi-static stretching, long wavelength modes are favored in the early stages of neck development for plates of both rate-independent materials and viscous materials. When the stretch rate is sufficiently large, 


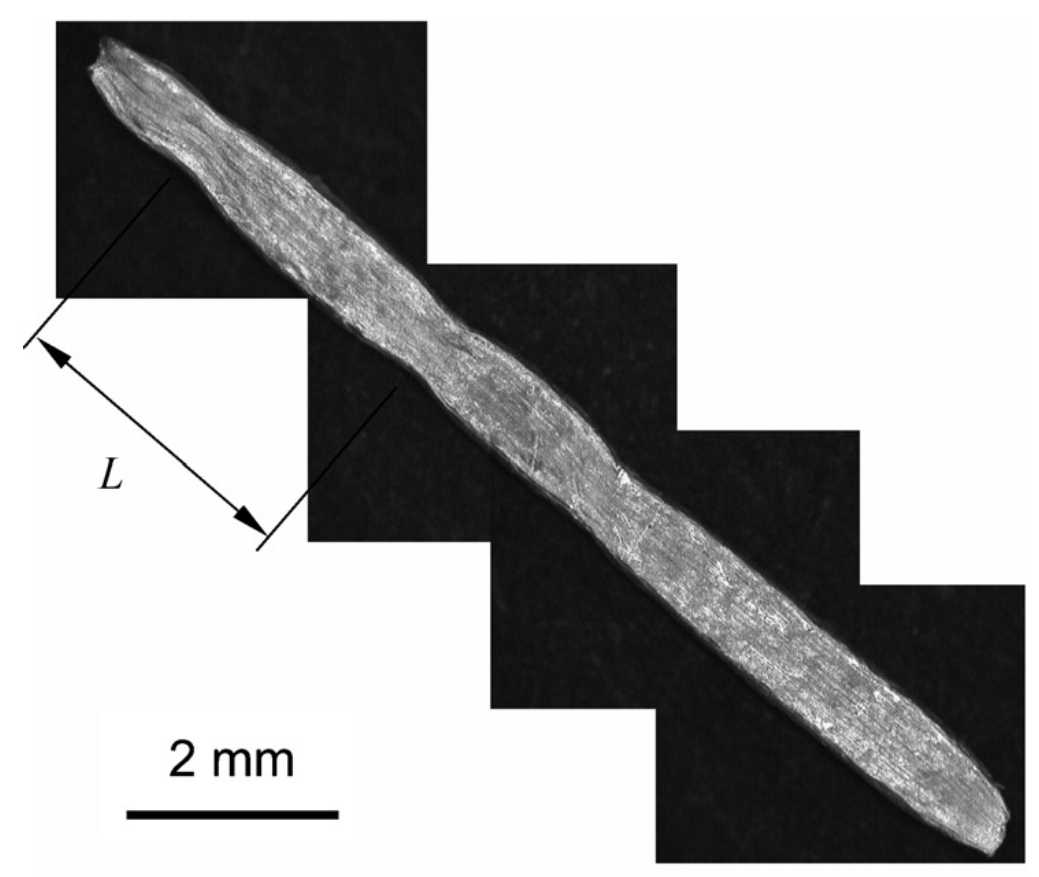

Fig. 6. Segment of an aluminum ring that has been dynamically expanded showing a necking mode with wavelengths several times the specimen width (Zhang and Ravi-Chandar, 2006). The cross-section of the ring is rectangular with initial thickness $0.5 \mathrm{~mm}$ and width $1 \mathrm{~mm}$. The width view is shown.

inertia becomes important and modes with finite wavelengths grow the fastest. This behavior is evident (Fig. 6) in extensive recent experiments on dynamic ring expansion conducted by Zhang and Ravi-Chandar (2006), building upon earlier ring expansion experiments of Niordson (1965) and Grady and Benson (1983). Fressengeas and Molinari (1994), Shenoy and Freund (1999), and Mercier and Molinari (2003) used perturbation analysis to study the relation among undulation growth, stretch-rate and undulation wavelength under incompressible plane strain stretching. Here we focus on the analysis of Shenoy and Freund, which generalizes that of Hill and Hutchinson to include inertia. The study examines the exponential growth rate of small undulation modes as a function of wavelength that are proportional to $\eta_{0} \mathrm{e}^{\mathrm{t} / t^{*}}$, where $\eta_{0}$ is a measure of the amplitude of the current undulation and $t^{*}$ is the growth time scale. The analysis identifies modes that grow the fastest in the early stage of necking, i.e. those with smallest $t^{*}$. The fundamental solution for the perfect plate about which the perturbation expansion is carried out is an exact dynamic solution with a uniform stretch-rate $\dot{\lambda}$. At the instant when the undulation growth rate is assessed, $\lambda=1$ such that the overall strain-rate is $\dot{\bar{\varepsilon}}=\dot{\lambda}$. The Shenoy-Freund analysis is carried out for a finite length plate with boundary conditions consistent with periodicity, but it can also be applied to an infinite plate with periodic modes which is the view taken here. The instantaneous moduli governing the plane strain increments of deformation are again $E_{\mathrm{t}}$ and $\mu$, and these do not evolve with time in the analysis. For the rate-independent power-law solid (1) with $m=0$ and $2 \mu / E_{\mathrm{t}}=1 / 2 N$, Shenoy and Freund obtained the stress, $\sigma$, and the wavelength, $L$, of the fastest growing sinusoidal mode with prescribed normalized growth time, $\dot{\lambda} t^{*}$. The result of this analysis is restated briefly in the Appendix emphasizing the dimensionless parameters relevant to the infinite plate with sinusoidal undulations: i.e. $\dot{\lambda} t^{*}, \dot{\lambda} h / \sqrt{E_{\mathrm{t}} / \rho}, \sigma / E_{\mathrm{t}}, h / L$ and $N$ where $\rho$ is the density and $h$ is the current average thickness. As emphasized by Shenoy and Freund, the normalized growth time, $\dot{\lambda} t^{*}$, represents the overall stretch increment that occurs in the time increment, $t^{*}$, during which the amplitude of the undulation increases by a factor e. The smaller is $\dot{\lambda} t^{*}$, the more rapid is the undulation growth relative to overall straining. Since $\sqrt{E_{\mathrm{t}} / \rho}$ is the plastic wave speed, the all-important inertia parameter, $\dot{\lambda} h / \sqrt{E_{\mathrm{t}} / \rho}$, represents the ratio of the relative transverse contraction velocity of the lateral surfaces of the plate to the plastic wave speed.

The plot in Fig. 7 presents the stress required to achieve a specified undulation growth time, $\dot{\lambda} t^{*}=0.071$, as a function of the inverse normalized undulation wavelength, $h / L$, for several values of $\dot{\lambda} h / \sqrt{E_{\mathrm{t}} / \rho}$, all with 


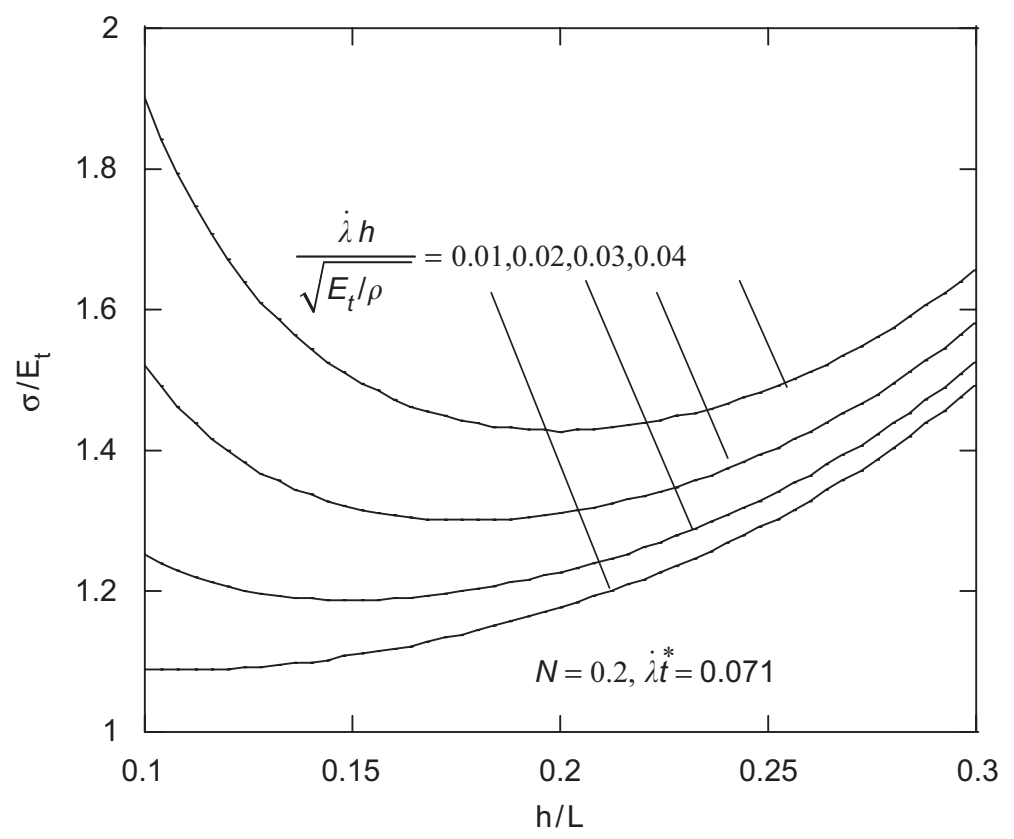

Fig. 7. Stress required to achieve the normalized undulation growth time, $\dot{\lambda} t^{*}=0.071$, under uniform dynamic stretching specified by $\dot{\lambda} h / \sqrt{E_{\mathrm{t}} / \rho}$, as determined by the analysis of Shenoy and Freund (1999). In the current state, $E_{\mathrm{t}}$ is the tangent modulus, $h$ is the plate thickness, and $L$ is the wavelength of the sinusoidal undulation. The results have been computed for a rate-independent power law material with strain hardening exponent $N$.

$N=0.2$. The lowest stress required to achieve this undulation growth rate is associated with a finite wavelength that scales with the plate thickness. The larger is $\dot{\lambda} h / \sqrt{E_{\mathrm{t}} / \rho}$, the shorter is the wavelength, but the minimum with respect to wavelength is quite flat implying that a relatively wide range of wavelengths will be competing for dominance in the early stages of growth. Fig. 8 presents results similar to those of Shenoy and Freund for combinations of wavelength and stress associated with the most rapidly growing undulation (all with $\dot{\lambda} t^{*}=0.071$ ), but expressed in terms of the dimensionless parameters relevant to the infinite plate. For specified $\dot{\lambda} h / \sqrt{E_{\mathrm{t}} / \rho}$, any mode with wavelength differing from that in Fig. 8A has a longer growth time than $\dot{\lambda} t^{*}=0.071$. The weak influence of the strain hardening exponent, $N$, is evident. Fig. 9 presents results for several normalized growth times.

The Shenoy-Freund analysis reveals that dynamic straining favors the early growth of finite wavelength undulations, with $L$ typically in the range, $3 h-10 h$, depending on $\dot{\lambda} h / \sqrt{E_{\mathrm{t}} / \rho}$ and $\dot{\lambda} t^{*}$. The elevation of stress above the stress at onset of quasi-static necking (i.e. $\sigma=E_{\mathrm{t}}$ ) strongly suggests neck retardation due to inertia at high stretch-rates. However, the perturbation analysis is only accurate in the early stages of undulation growth before well-developed necks form, and thus the strain associated with the stress in Figs. 7-9 is not an accurate reflection of the full retardation effect. Guduru and Freund (2002) closely examined behavior at very high stretch rates in a range of $\dot{\lambda} h / \sqrt{E_{\mathrm{t}} / \rho}$ higher than that considered here. They found that at sufficiently high stretch rates the fastest growing wavelengths become shorter than the thickness of the strip and the mode has the character of a surface instability.

In the sections of the paper which follow, the simple two-state model of Section 1.1 is first extended to include inertia and then applied to study dynamic neck retardation. Then, an extensive finite element computational study is carried out with the aim of providing unifying results under finite strain for fully developed necks.

\section{Two-state model for dynamic neck development}

There have been a number of attempts to capture the effect of inertia on neck development using simplified one-dimensional models (Regazzoni et al., 1986; Tuggu et al., 1990). Here, a two-state model is 

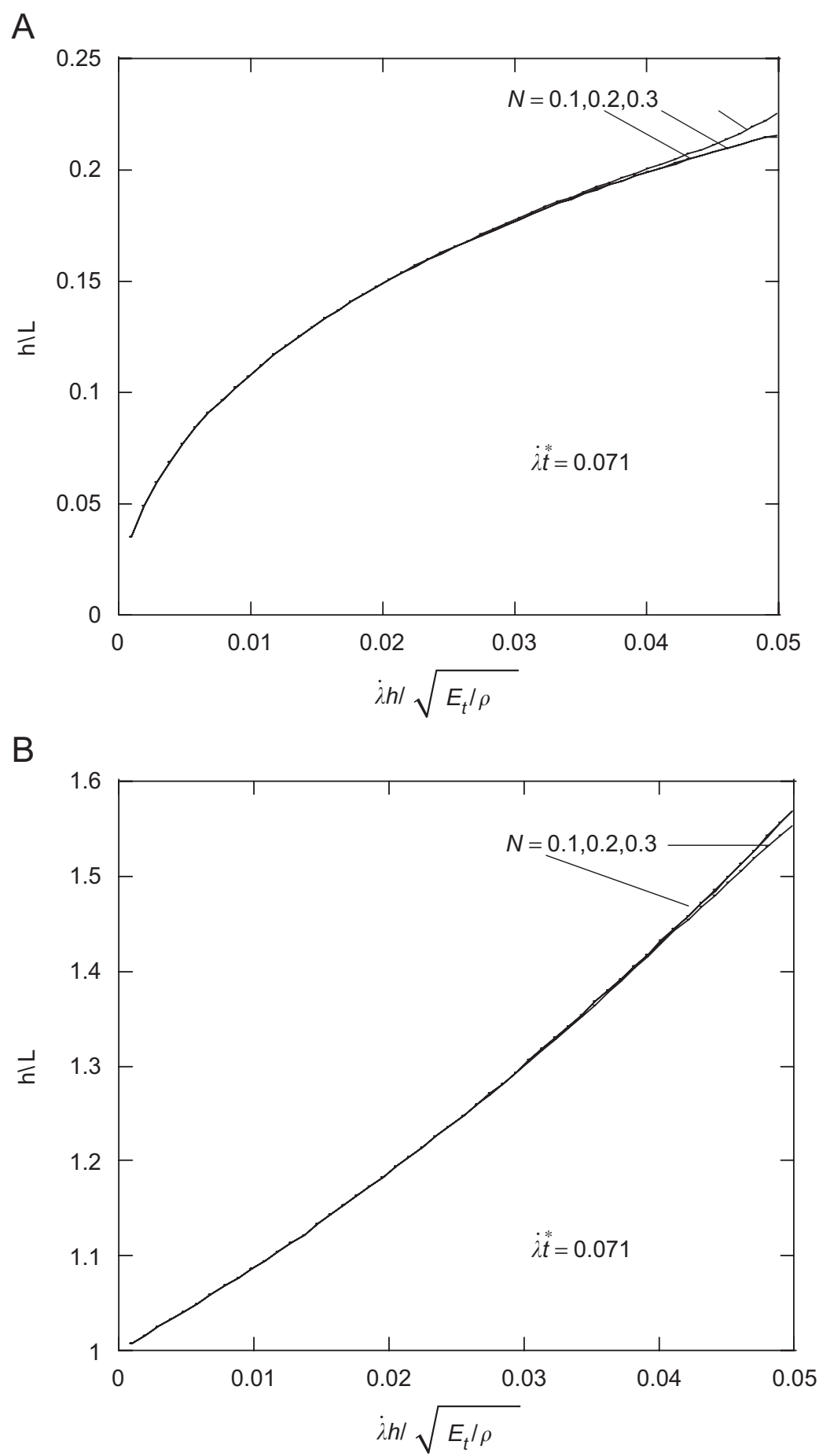

Fig. 8. Undulation wavelength (A) associated with the minimum stress (B) required to achieve the undulation growth time, $\dot{\lambda} t^{*}=0.071$ under uniform dynamic stretching specified by $\dot{\lambda} h / \sqrt{E_{\mathrm{t}} / \rho}$, as determined by the analysis of Shenoy and Freund (1999). In the current state, $E_{\mathrm{t}}$ is the tangent modulus, $h$ is the plate thickness, and $L$ is the wavelength of the sinusoidal undulation.

introduced that identifies the essential dimensionless parameters and accurately captures most trends in neck retardation as dependent on material properties and inertia. The initial geometry and the geometry in the current deformed configuration assumed for the dynamic two-state model are given in Fig. 10. In addition to assuming incompressible plane strain deformations, two approximations are made: (i) each of the segments, A and B, are states of uniform stress and strain. (ii) The stress state in each segment is uniaxial plane strain-effects due to transverse stress triaxiality are ignored. Otherwise, the equations which 


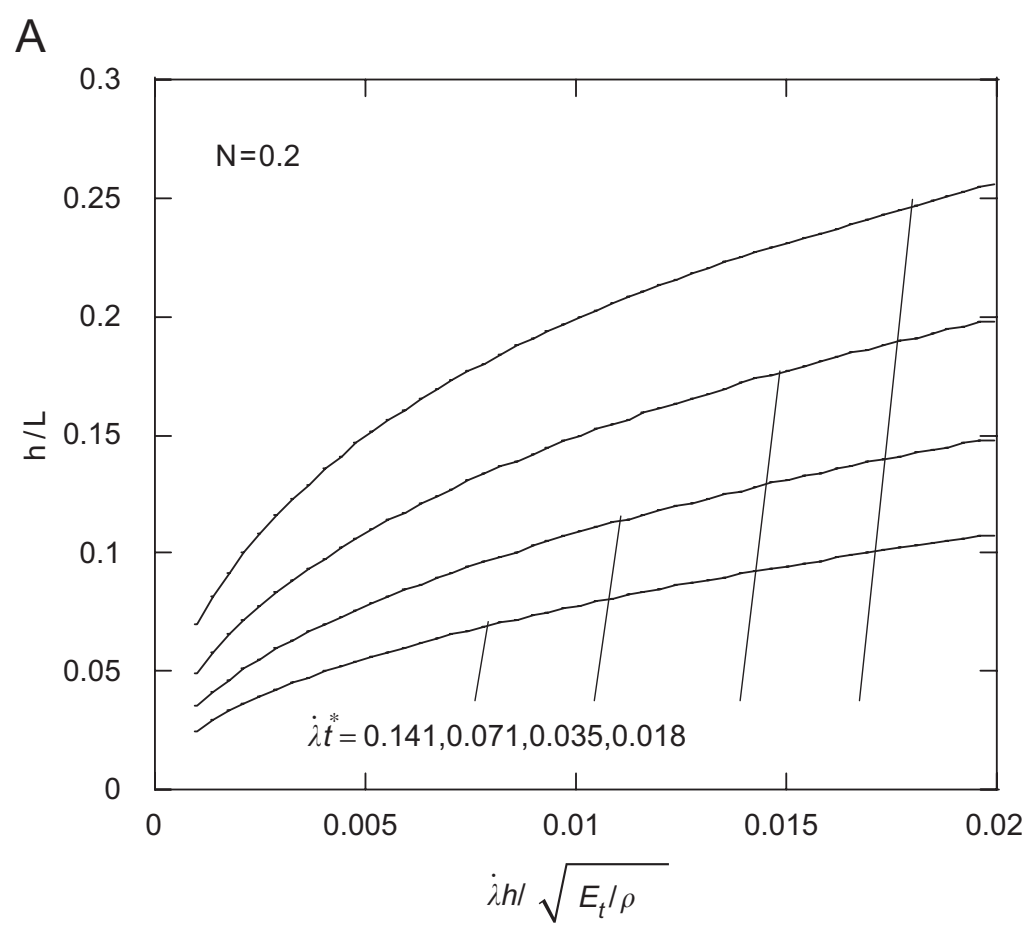

B

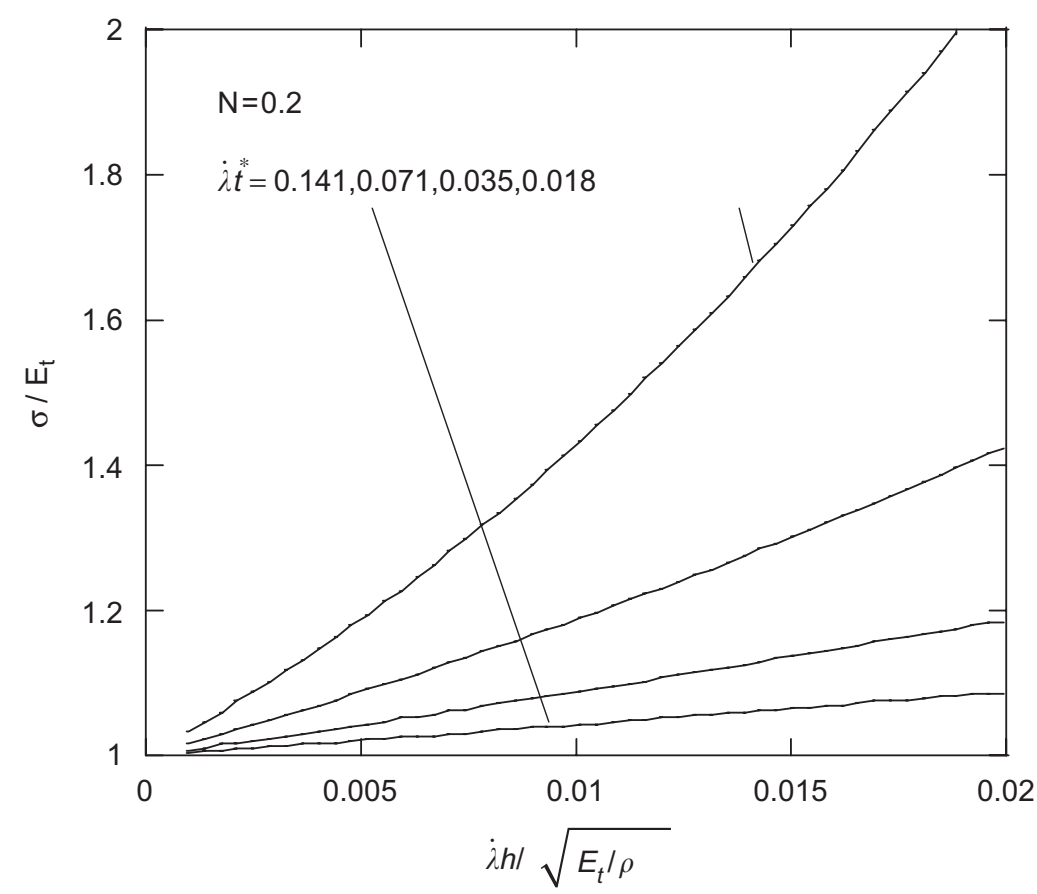

Fig. 9. Undulation wavelength (A) associated with the minimum stress (B) required to achieve four undulation growth times, $\dot{\lambda} t^{*}$, under uniform dynamic stretching specified by $\dot{\lambda} h / \sqrt{E_{\mathrm{t}} / \rho}$, as determined by the analysis of Shenoy and Freund (1999). In the current state, $E_{\mathrm{t}}$ is the tangent modulus, $h$ is the plate thickness, and $L$ is the wavelength of the sinusoidal undulation.

follow exactly account for finite geometry changes, nonlinear stress-strain behavior and dynamic equilibrium. In standard notation, denote the overall stretch by $\lambda=L / L^{0}$ and overall logarithmic strain by $\bar{\varepsilon}=\ln (\lambda)$, with similar notation for each segment. An ordinary differential equation relating the evolution of the stress and strain in segment $\mathrm{A}$ to that in segment B, is obtained based on approximations (i) and (ii) noted above as follows. Invoking (i) and (ii), integrate the equilibrium equation in the current state over one complete periodic unit of the plate in Fig. 10. It is straightforward to show that the results 


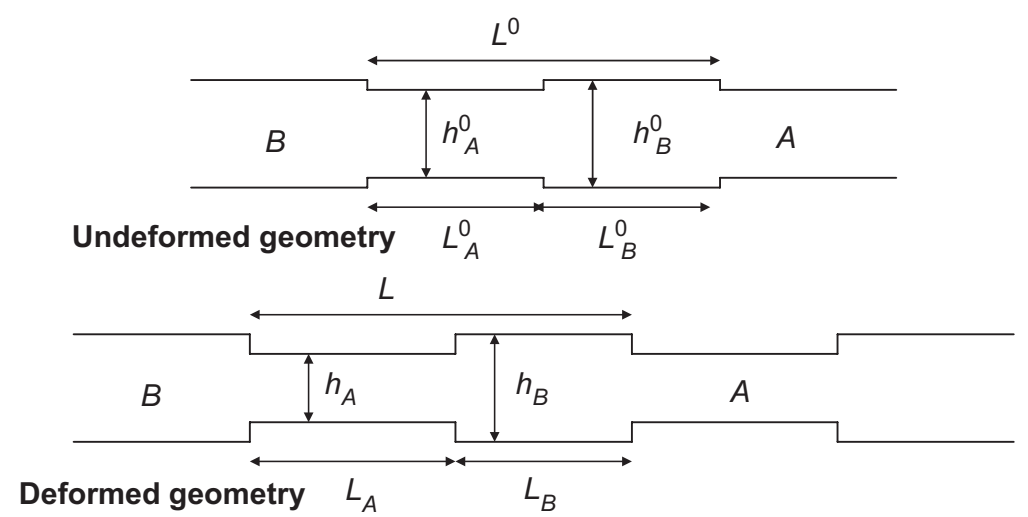

Fig. 10. Undeformed and deformed geometry of the two-state model for dynamic neck growth under overall stretching.

can be written as

$$
\begin{aligned}
\frac{1}{\sigma_{R}} & {\left[\sigma_{B} \mathrm{e}^{-\varepsilon_{B}}-\left(1-\eta_{0}\right) \sigma_{A} \mathrm{e}^{-\varepsilon_{A}}\right] } \\
= & \frac{1}{2}\left(\frac{L_{B}^{0}}{h_{B}^{0}}\right)^{2}\left\{\left[\left(\left(1-\eta_{0}\right) R^{2}+2 R\right)\right] \mathrm{e}^{\varepsilon_{A}}\left(\frac{\mathrm{d}^{2} \varepsilon_{A}}{\mathrm{~d}^{2} \tau}+\left(\frac{\mathrm{d} \varepsilon_{A}}{\mathrm{~d} \tau}\right)^{2}\right)+\mathrm{e}^{\varepsilon_{B}}\left(\frac{\mathrm{d}^{2} \varepsilon_{B}}{\mathrm{~d}^{2} \tau}+\left(\frac{\mathrm{d} \varepsilon_{B}}{\mathrm{~d} \tau}\right)^{2}\right)\right\} .
\end{aligned}
$$

Here, $\sigma_{R}$ is any conveniently chosen reference stress,

$$
R=\frac{L_{A}^{0}}{L_{B}^{0}}, \quad \eta_{0}=1-\frac{h_{A}^{0}}{h_{B}^{0}},
$$

and the dimensionless time variable is

$$
\tau=\frac{t \sqrt{\sigma_{R} / \rho}}{h_{B}^{0}} .
$$

The overall stretch is related to the stretches in the individual segments by $\lambda=\left(R \lambda_{A}+\lambda_{B}\right) /(1+R)$. For the case considered here, where the overall stretch-rate, $\dot{\lambda}$, is prescribed to be constant, (4) reduces to

$$
\frac{\mathrm{d}^{2} \lambda_{A}}{\mathrm{~d} \tau^{2}}=\frac{2\left(h_{B}^{0} / L^{0}\right)^{2}(1+R)^{2}}{\left(\left(1-\eta_{0}\right) R^{2}+R\right)} \frac{1}{\sigma_{R}}\left[\sigma_{B} \mathrm{e}^{-\varepsilon_{B}}-\left(1-\eta_{0}\right) \sigma_{A} \mathrm{e}^{-\varepsilon_{A}}\right]
$$

supplemented by $\lambda_{B}=(1+R) \lambda-R \lambda_{A}$. When inertia is negligible, $\mathrm{d}^{2} \lambda_{A} / \mathrm{d} \tau^{2} \approx 0$ such that (7) reduces to the equation governing the quasi-static two-state model: $\sigma_{B} h_{B}=\sigma_{A} h_{A}$.

Two uniaxial constitutive models will be considered: the power-law solid (1) introduced earlier, and another widely used relation called the over-stress relation:

$$
\sigma=\sigma_{R} \varepsilon^{N}\left(1+\left(\dot{\varepsilon} / \dot{\varepsilon}_{R}\right)^{\mathrm{p}}\right)
$$

with $\mathrm{p}$ and $\dot{\varepsilon}_{R}$ as parameters characterizing the material rate-dependence. It can be noted immediately that the dimensionless parameters characterizing the imposed stretch-rate and the material rate-dependence (in addition to $m$ or $\mathrm{p}$ ) can be taken as

$$
\frac{\dot{\lambda} h_{B}^{0}}{\sqrt{\sigma_{R} / \rho}} \text { and } \frac{\dot{\varepsilon}_{R} h_{B}^{0}}{\sqrt{\sigma_{R} / \rho}} \text {. }
$$

In the perturbation studies $E_{\mathrm{t}}$ was used in the dimensionless stretch rate since it is held fixed in assessing small undulation growth. In the finite strain problems, the dimensionless parameters in (9) employ $\sigma_{R}$ rather than $E_{\mathrm{t}}$ because the tangent modulus changes as the neck develops.

Numerical simulations based on the simple dynamic two-state model (7) for uniform overall stretching are readily carried out. The model does a good job of capturing essentially all the important trends except for 
identifying the critical wavelength. Because stress triaxiality is ignored, the model exhibits no tendency to suppress short wavelength modes which give rise to high triaxiality. However, as long as realistic values for $L^{0} / h_{B}^{0}$ and $R=L_{A}^{0} / L_{B}^{0}$ are used, trends with respect to the other parameters are correctly reproduced, as will be illustrated by comparing with selected finite element simulations. In this connection it is relevant to observe that results from the two-state model do not depend on either $L^{0} / h_{B}^{0}$ or $R=L_{A}^{0} / L_{B}^{0}$ in the inertia-free limit. In the results presented below we have chosen, $L^{0} / h_{B}^{0}=3$ and $R=0.5$. Necking is assumed to occur when either $\mathrm{d} \varepsilon_{B} / \mathrm{d} \tau=0$ or $\varepsilon_{A}=5$, whichever is attained first. It will be demonstrated that the choice $\varepsilon_{A}=5$, which is arbitrary, does not have an important effect on the general trends.

The combined role of material rate-dependence and inertia on neck retardation was emphasized by Needleman (1991) based on dynamic finite element simulations. The advantage of the two-state model is that it can efficiently generate results over the full range of the parameter space, as will be illustrated for selected trends. The role of material strain-rate dependence for the overstress relation (8) is illustrated in Fig. 11 under conditions of dynamic stretching. Fig. 11A presents the overall strain at necking, $\bar{\varepsilon}=\ln \left(L / L^{0}\right)$, while Fig. 11B presents the strain in segment $\mathrm{B}$ at necking, $\varepsilon_{B}=\ln \left(L_{B} / L_{B}^{0}\right)$. Because the strain in segment $\mathrm{A}$ is always larger than that in $\mathrm{B}, \bar{\varepsilon}$ is always larger than $\varepsilon_{B}$. Nevertheless, the difference in these two strains is not large at necking and either can be used to display trends of interest here. The main trend evident in Fig. 11 is the substantial necking retardation due to both inertial effects, as measured by $\dot{\lambda} h_{B}^{0} / \sqrt{\sigma_{R} / \rho}$, and material rate-dependence as measured by $\dot{\varepsilon}_{R} h_{B}^{0} / \sqrt{\sigma_{R} / \rho}$. When these two dimensionless parameters are of comparable magnitude, each makes comparable contributions to neck retardation as will be discussed further in the next section.

The role of the strain-rate hardening exponent, $m$, in the power-law relation (1) in combination with dynamic stretching is presented in Fig. 12. In the power-law relation, the strain-rate amplifies the stress over the entire strain-rate range, while for the overstress relation the stress is only amplified in the range of high strain-rates. This accounts for the fact that significant neck retardation is observed over the entire range of $\dot{\lambda} h_{B}^{0} / \sqrt{\sigma_{R} / \rho}$ for the power law material, as would be expected from Fig. 3 where inertial effects are absent. Fig. 12 also illustrates that both material rate-dependence and inertial effects combine to significantly increase the strain prior to necking.

Han and Tvergaard (1995) have noted that imperfections play an essential role in dynamic instability problems such as necking and have computed results displaying the effect of imperfection amplitude on necking. These authors have also studied the effect of imperfection amplitude and shape on neck development in dynamically expanding circular rings. As emphasized in the Introduction, a flat plate with neither geometric nor material imperfections will not exhibit necking deformations under dynamic stretching. Moreover, the

A

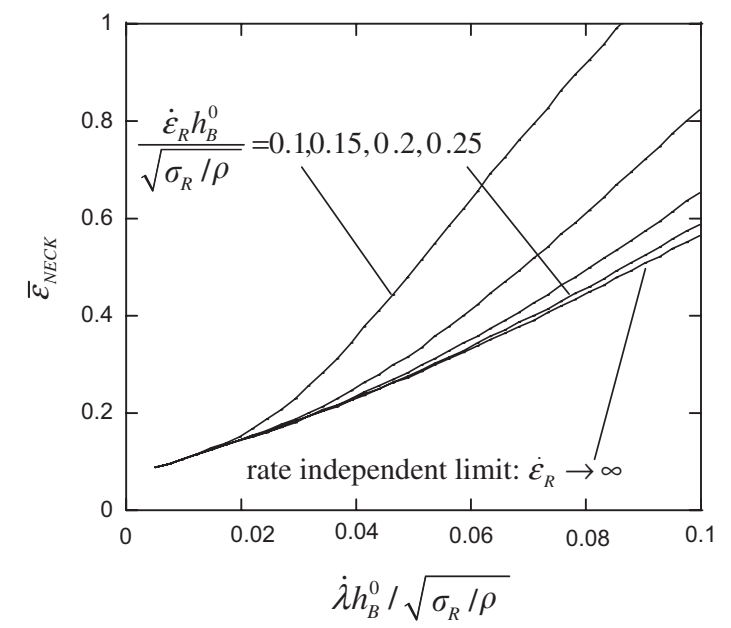

B

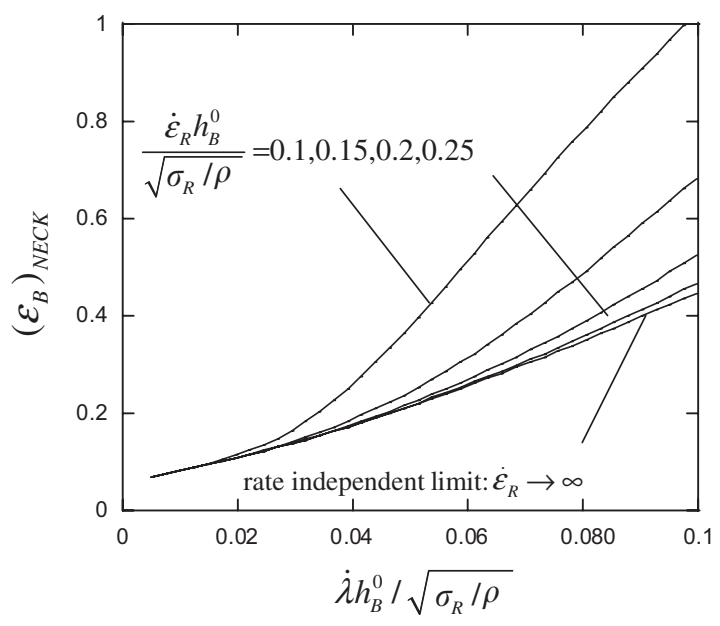

Fig. 11. Necking strain as a function of normalized stretch-rate for several values of the material rate-dependence parameter, $\dot{\varepsilon}_{R} h_{B}^{0} / \sqrt{\sigma_{R} / \rho}$, including the rate-independent limit, for the overstress relation (8). The parameters specifying the model are $N=0.1, p=5$, $L^{0} / h_{B}^{0}=3, L_{A}^{0} / L_{B}^{0}=0.5$ and $\eta_{0}=0.01$. The overall strain at necking is plotted in (A) and the strain in segment B at necking is plotted in (B). 


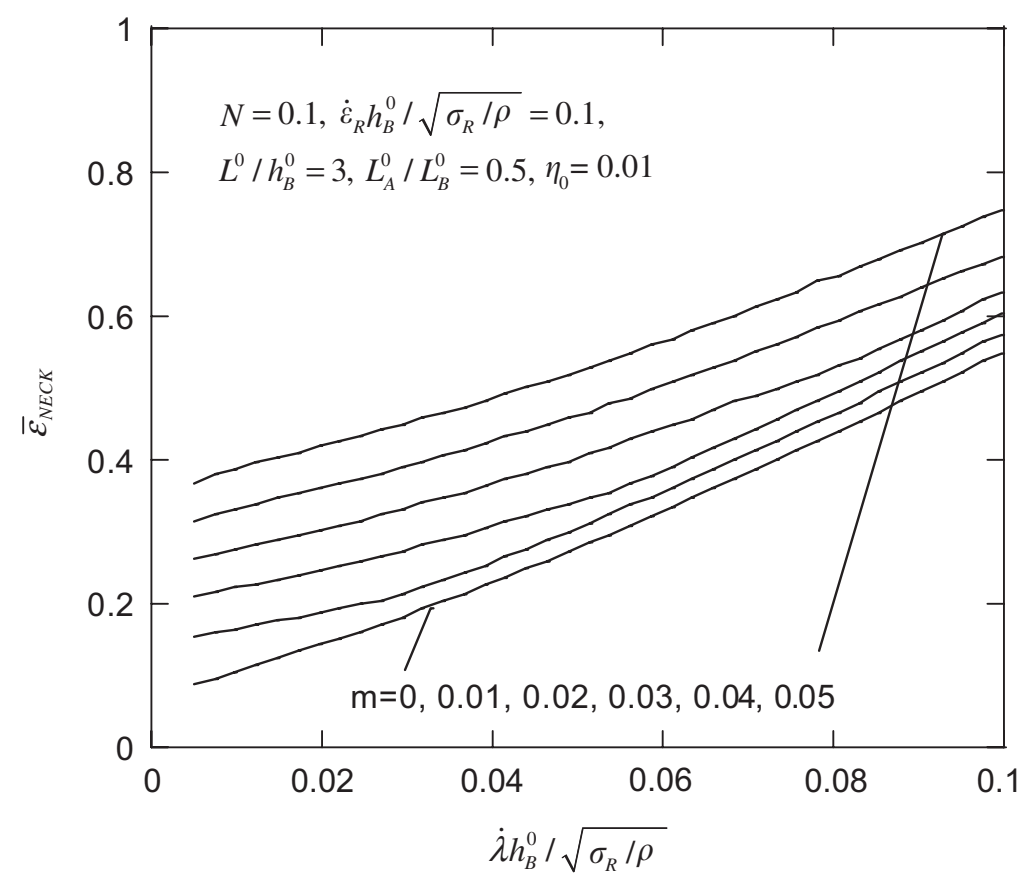

Fig. 12. The overall strain at necking as a function of normalized stretch-rate for several values of the material rate-dependence parameter, $m$, including the rate-independent limit, for the power-law relation (1). The dependence on $\dot{\varepsilon}_{R} h_{B}^{0} / \sqrt{\sigma_{R} / \rho}$ is weak.

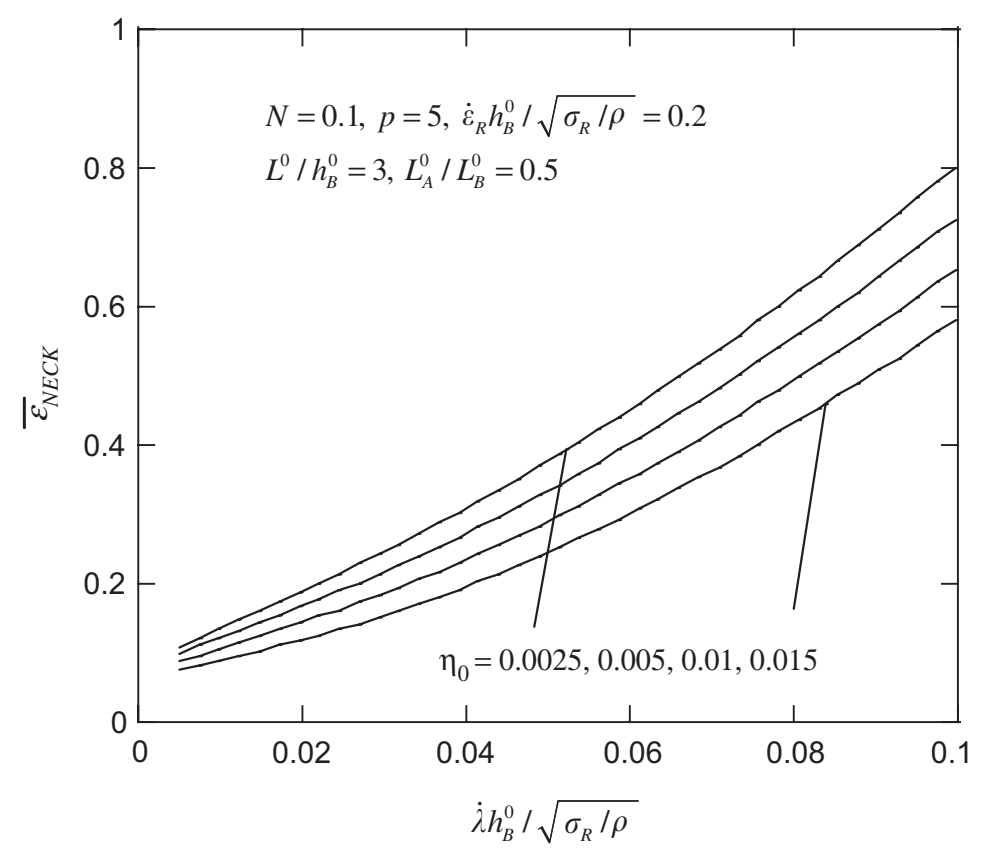

Fig. 13. The overall strain at necking as a function of normalized stretch-rate for several values of the initial geometric imperfection, $\eta_{0}$, for the overstress relation (8).

early growth of undulations is linearly proportional to the amplitude of the initial imperfection. Fig. 13 displays the dependence of the overall necking strain from the two-state model on the dynamic stretch rate parameter for different levels of geometric imperfection, $\eta_{0}$, for the overstress relation. The relation between neck retardation and the imperfection amplitude is not linear, but the necking strain becomes unbounded as the imperfection amplitude goes to zero. 


\section{Computational model for assessing necking under dynamic stretching}

\subsection{Computational model and procedures}

Dynamic stretching of an infinite plate under plane strain constraint, $\dot{\varepsilon}_{R} \rightarrow \infty$, is considered with the undeformed geometry shown in Fig. 14. Initial and boundary conditions are imposed on the unit periodic cell in Fig. 14 consistent with a constant stretching rate. The reference configuration is an infinite imperfection-free plate of initial thickness $h^{0}$. The imperfect plate has an initial sinusoidal geometric imperfection with initial wavelength, $L^{0}$, and amplitude, $\eta_{0}$, as depicted in Fig. 14,

$$
h=h^{0}\left(1-\frac{1}{2} \eta_{0} \cos \frac{2 \pi X_{1}}{L_{0}}\right) .
$$

Cartesian coordinates $\left(X_{1}, X_{2}\right)$ mark the location of a material point in the undeformed state, while the location of the same material point in the deformed state is $\left(x_{1}, x_{2}\right)$. With reference to Fig. 14, attention will be restricted to solutions that are periodic in the $X_{1}$-direction with period $\lambda L_{0}$ in the current state, where $\lambda$ is the overall imposed stretch. The solution is also required to be symmetric with respect to the center-line $X_{2}=x_{2}=0$ and about the mid-point of the periodic unit at $X_{1}=0$. Thus, invoking a Lagrangian description referred to the undeformed state, only the upper-right quarter of the section for $X_{1} \geqslant 0$ and $X_{2} \geqslant 0$ in Fig. 14 needs to be considered. The tractions on the top of this cell are taken to be zero, while the bottom of the cell has zero displacement in the $X_{2}$-direction and zero shear traction. The left end of the section at $X_{1}=0$ has zero horizontal displacement and zero shear traction. The right end at $X_{1}=L_{0} / 2$ also has zero shear traction, and it is moved horizontally with constant velocity $V_{0} / 2$ such that the length of the periodic unit at any time, $t$, is $L=L_{0}+V_{0} t$. With $\lambda=L / L_{0}$ as the overall stretch, the overall stretch-rate, $\dot{\lambda}=V_{0} / L_{0}$, is prescribed to be constant, but the rate of the overall logarithmic strain, $\bar{\varepsilon}=\ln (\lambda)$, diminishes in time as $\dot{\bar{\varepsilon}}=\dot{\lambda} / \lambda$. Initial conditions will be described below.

Shenoy and Freund (1999) showed that if one neglects compressibility and if the plate is uniform, the plane strain solution for uniform stretching $\left(\dot{\lambda}=V_{0} / L_{0}, \ddot{\lambda}=0\right)$,

$$
x_{1}=\lambda X_{1}, x_{2}=\lambda^{-1} X_{2},
$$

is an exact solution to the dynamic field equations. The velocity and acceleration components are

$$
\begin{gathered}
v_{1}=\dot{\lambda} X_{1}=(\dot{\lambda} / \lambda) x_{1}, v_{2}=-\lambda^{-2} \dot{\lambda} X_{2}=-(\dot{\lambda} / \lambda) x_{2}, \\
a_{1}=\ddot{\lambda} X_{1}=0, a_{2}=2 \lambda^{-3} \dot{\lambda}^{2} X_{2}=2 \lambda^{-2} \dot{\lambda}^{2} x_{2} .
\end{gathered}
$$

The stress component, $\sigma_{22}$, is determined directly from equilibrium as

$$
\sigma_{22}\left(x_{2}\right)=\rho(\dot{\lambda} / \lambda)^{2}\left(x_{2}^{2}-(h / 2)^{2}\right)
$$

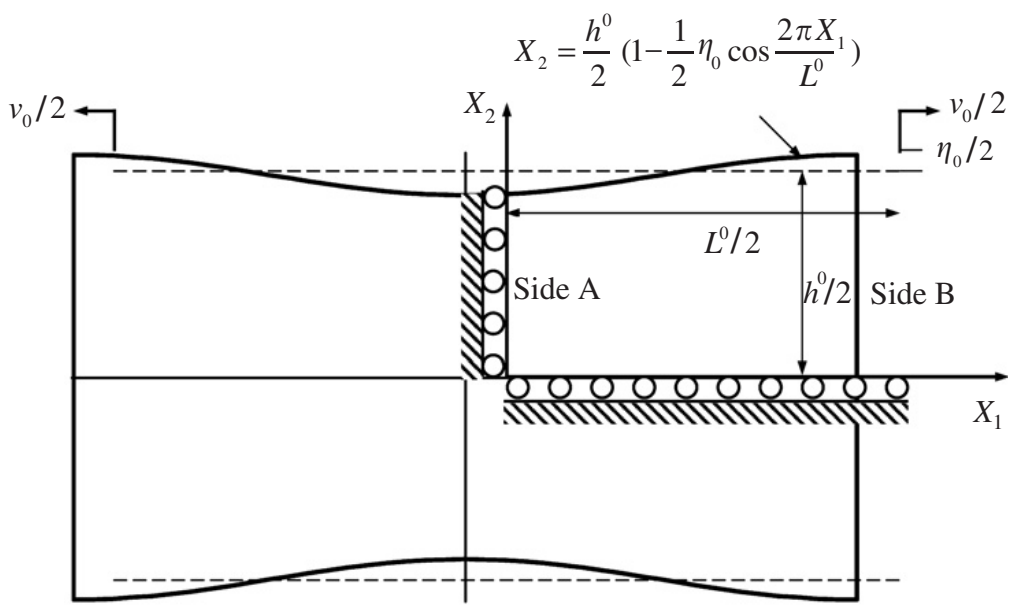

Fig. 14. Schematic diagram of one periodic section of metal plate under high strain-rate extension. 
where $\rho$ is the density and $h$ is the current thickness of the plate. The other nonzero stress components, $\sigma_{11}$ and $\sigma_{33}$, are independent of $x_{1}$ and can be determined with the aid of the constitutive relation. This fundamental solution for the imperfection-free plate has stresses and strains with no dependence on $x_{1}$.

The fundamental solution is used to impose initial conditions on the dynamic problem. Specifically, at the start, at $t=0$, the displacements are zero and the velocities are given by (12) with $\lambda=1$. These initial conditions are not exact for the problem posed in this paper for two reasons, but the errors are small. (i) The constitutive model introduced below has elastic compressibility. (ii) The solution (12) applies strictly only to the perfect plate whereas, in the solved problems, a small initial imperfection is always present. It should be noted that it is essential to identify the correct initial conditions in modeling behavior consistent with a long plate under uniform stretching. For example, if the right end of the unit at $\mathrm{A}$ is abruptly moved at $t=0$ with velocity $V_{0}$ while the remainder of the plate is initially at rest, a wave is initiated that propagates from $A$ to $B$ (Needleman, 1991). For sufficiently intense waves, the plastic wave itself can serve as the trigger for inducing a neck.

The problem is posed within a finite strain framework accounting for large geometry changes. The stresses and strains used below are true stresses and logarithmic strains. The constitutive behavior of the material metal is elastic/visco-plastic. The elasticity is isotropic with modulus $E$ and Poisson's ratio $v$ and the material density is $\rho$. Uniaxial tensile behavior is characterized by the over-stress relation augmented by an initial elastic response

$$
\begin{aligned}
\sigma & =E \varepsilon, \quad \varepsilon \leqslant \varepsilon_{Y} \equiv \sigma_{Y} / E \\
\sigma & =\sigma_{Y}\left(\frac{\varepsilon}{\varepsilon_{Y}}\right)^{N}\left[1+\left(\dot{\varepsilon}^{\mathrm{p}} / \dot{\varepsilon}_{R}\right)^{\mathrm{p}}\right]=\sigma_{R} \varepsilon^{N}\left[1+\left(\dot{\varepsilon}^{\mathrm{p}} / \dot{\varepsilon}_{R}\right)^{\mathrm{p}}\right], \quad \varepsilon>\varepsilon_{Y}
\end{aligned}
$$

where $\sigma$ is the true stress, $\dot{\varepsilon}^{\mathrm{p}}$ is the logarithmic effective plastic strain-rate defined as $\dot{\varepsilon}^{\mathrm{p}}=\sqrt{2 \dot{\varepsilon}_{i j}^{\mathrm{p}} \dot{\varepsilon}_{i j}^{\mathrm{p}} / 3}$, $\sigma_{R}=\sigma_{Y} \varepsilon_{Y}^{-N}, N$ is the strain hardening exponent, and the two constants specifying the material rate sensitivity are $\dot{\varepsilon}_{R}$ and p The standard Mises flow law is assumed: $\dot{\varepsilon}_{i j}^{\mathrm{p}}=3 \dot{\varepsilon}^{\mathrm{p}} s_{i j} / 2 \sigma_{e}$ where $s_{i j}$ is the stress deviator and $\sigma_{e}=\sqrt{3 s_{i j} s_{i j} / 2}$.

The computational problem defined above was solved using the finite element method as available in ABAQUS Explicit (2004). Four-node bilinear plane strain elements with reduced integration are used. For all cases, near-square elements (elements are distorted slightly due to the imposed initial imperfection) with approximate size $H_{0} / 40$ are uniformly distributed over the unit computational cell, corresponding to 20 elements uniformly distributed through the half-thickness of the plate. Computational convergence was established with this meshing scheme by employing a series of similar meshes of various refinements. As described above, a constant, uniform horizontal velocity $V_{0} / 2$ is applied to the right end of the unit cell, and the initial velocity condition from (12) is imposed along with zero displacements at $t=0$.

\subsection{The effect of material and inertial effects on neck retardation}

Features of neck evolution are illustrated in Fig. 15 for five imperfection wavelengths with the same imperfection amplitude, $\eta_{0}=0.04$, and subject to the same overall stretch rate, $\dot{\lambda} h^{0} / \sqrt{\sigma_{R} / \rho}=0.09$. The rateindependent limit $\left(\dot{\varepsilon}_{R} \rightarrow \infty\right)$ of the over-stress relation (14) is used. Fig. 15B displays evolution of the average strain at the minimum section of the neck, $\bar{\varepsilon}_{A} \cong \ln \left(h_{A}^{0} / h_{A}\right)$, as a function of the overall strain, $\bar{\varepsilon}=\ln (\lambda)$, while Fig. $15 \mathrm{~A}$ presents the corresponding strain evolution at the section of maximum thickness at B, i.e. $\bar{\varepsilon}_{B} \cong$ $\ln \left(h_{B}^{0} / h_{B}\right)$ vs. $\bar{\varepsilon}$. Fig. $15 \mathrm{C}$ shows the deformed shape at $\bar{\varepsilon}=0.37$ for each of the five imperfection wavelengths. Localization sets in at the overall strain when the strain at the thickest section ceases to increase. Beyond this point straining is localized with essentially all deformation occurring within the neck region. In the results which follow, the overall strain at necking, $\bar{\varepsilon}_{\mathrm{NECK}}$, is defined to be the overall strain at the point where the maximum strain in the thickest section is attained, as indicated by the dashed vertical lines in Fig. 15A. Note from Fig. 15 that the neck at $\mathrm{A}$ is in the early stage of development with this definition, and $\bar{\varepsilon}_{A}$ is not much larger than $\bar{\varepsilon}_{B}$ and $\bar{\varepsilon}_{\mathrm{NECK}}$. 


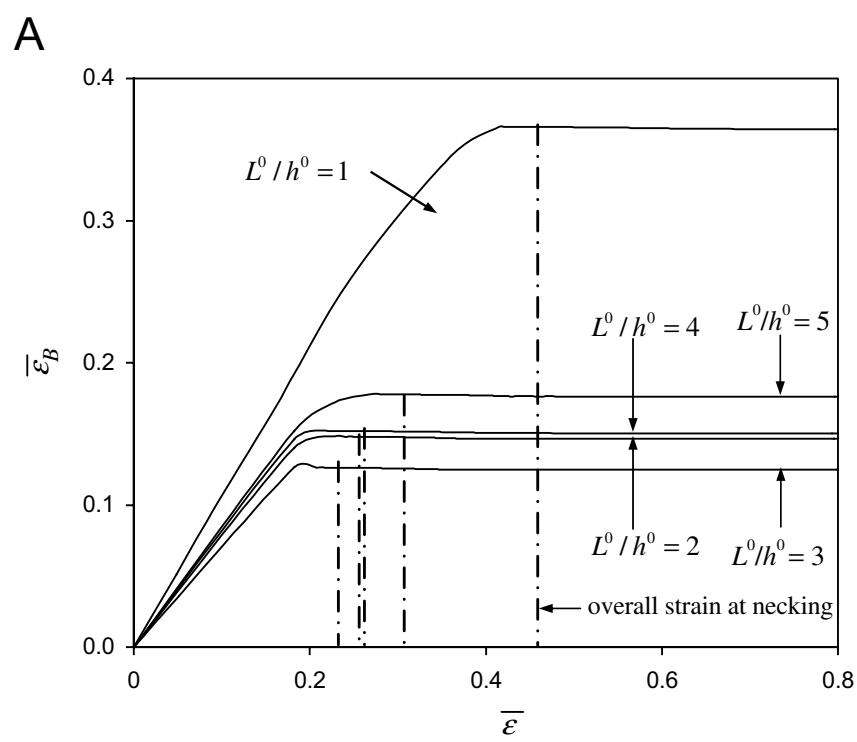

B
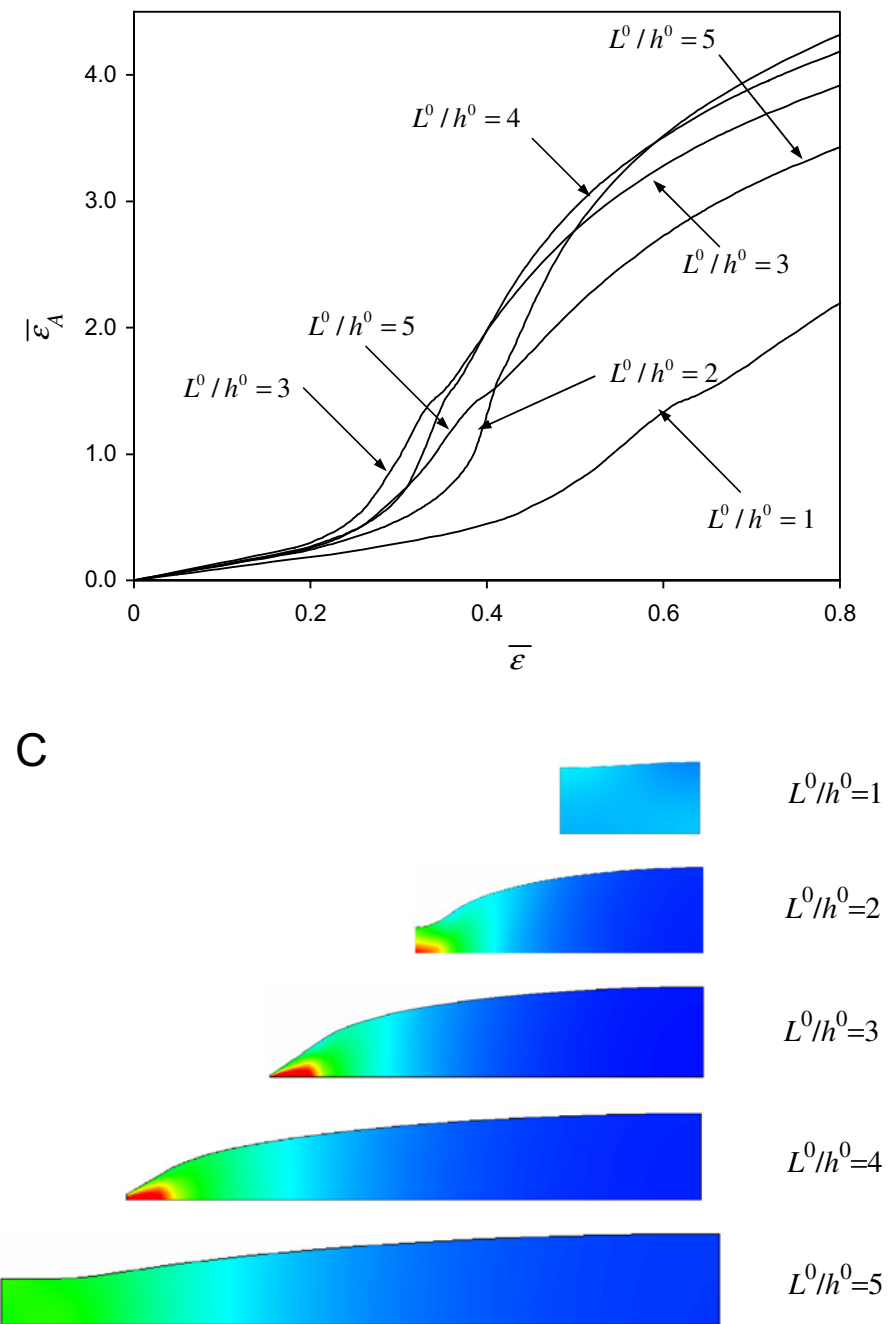

Fig. 15. (A) Average strain through the thickness at the thickest section (B) as a function of reference overall strain. (B) Average strain through the thickness at the thinnest section (A) as a function of reference overall strain. (C) Deformed configuration of plates, in each case at an overall strain, $\bar{\varepsilon}=0.37$. The plates are subjected to dynamic stretching with $\dot{\lambda} h^{0} / \sqrt{\sigma_{R} / \rho}=0.09$. Five imperfection wavelength ratios are considered: $L^{0} / h^{0}=1,2,3,4$, and 5 respectively. The imperfection amplitude is $\eta_{0}=0.04$ for all cases. The material has a hardening exponent of $N=0.1$ and $\sigma_{R} / E=0.002$. Material strain-rate dependence is not incorporated. For a steel plate $\left(\rho=8000 \mathrm{~kg} / \mathrm{m}^{3}\right)$ with initial thickness, $h^{0}=0.02 \mathrm{~m}$, and $\sigma_{R}=400 \mathrm{MPa}$, the overall stretch rate is $\dot{\lambda}=1000 \mathrm{~s}^{-1}$. 
The effect of the normalized imperfection wavelength, $L^{0} / h^{0}$, is apparent. Relatively little neck development occurs over the range of overall strain plotted in Fig. 15 for either the very short wavelength $\left(L^{0} / h^{0}=1\right)$ or the longest wavelength $\left(L^{0} / h^{0}=5\right)$, while significant neck development occurs for imperfection wavelengths in the range $2 \leqslant L^{0} / h^{0} \leqslant 4$ with the most advanced neck associated with $L^{0} / h^{0} \approx 3$ in this example. The necking strains, $\bar{\varepsilon}_{\text {NECK }}$, are presented in Fig. 16 as a function of normalized imperfection wavelength for various overall stretch rates. These results have also been computed assuming no material rate-dependence.

For the lowest two overall stretch rates, which are effectively only just above quasi-static, long wavelength imperfections are the most deleterious but there is little dependence on wavelength if $L^{0} / h^{0} \geqslant 3$. Over the range of overall stretch rates plotted in Fig. 16, the minimum necking strain is very close to that associated with the wavelength, $L^{0} / h^{0}=3$. Fig. 17 presents the minimum necking strain, $\bar{\varepsilon}_{\mathrm{NECK}}$, as a function of the dimensionless stretch rate for the wavelength $L^{0} / h^{0}=3$ for three values of the strain hardening exponent, computed with imperfection amplitude $\eta_{0}=0.04$. Unlike the early growth rate of the undulation in Fig. 7 which is essentially independent of $N$, the finite amplitude analysis of necking indicates a strong dependence on $N$ similar to what one would expect from the Considère result, $\bar{\varepsilon}_{\mathrm{NECK}}=N$, for quasi-static straining of rate-independent powerlaw materials with no imperfections. The reduction of the necking strain below $\bar{\varepsilon}_{\mathrm{NECK}}=N$ at low values of overall stretch rate when quasi-static behavior is approached is due to the initial imperfection. The necking strain is virtually independent of the elastic modulus. Results for $N=0.1$ in Fig. 17 were computed for three values of $\sigma_{R} / E$ with essentially identical results as evident in that figure.

The interplay between material rate-dependence and inertia in the phenomenon of neck retardation is brought out in Fig. 18 where the necking strain has been computed over a range of imperfection wavelengths for material properties representative of 304 stainless steel. Three sets of calculations have been performed: (1) with material rate-dependence but no inertia, (2) with inertia but no material rate-dependence $\left(\dot{\varepsilon}_{R} \rightarrow \infty\right)$, and (3) with both inertia and material rate-dependence. This example illustrates that both inertia and strainrate hardening contribute in a significant way to neck retardation in a manner that is not additive. Material rate-dependence has little influence on the critical wavelength associated with the minimum necking strainwhich appears to be determined primarily by inertia - but it can significantly increase the necking strain.

The curves in Fig. 19 display the role of material rate-dependence in combination with inertia on neck retardation for material behavior characterized by the overstress relation (14). The plot gives the nearminimum necking strain (associated with $L^{0} / h^{0}=3$ ) as a function of the dimensionless stretch-rate,

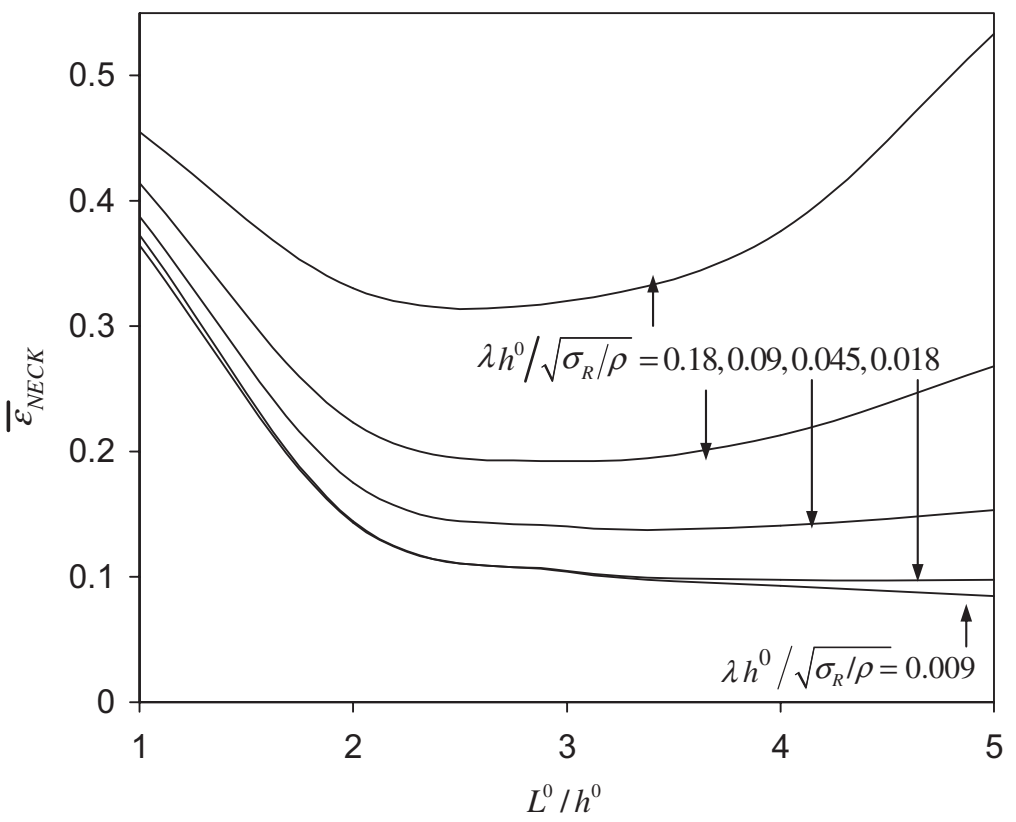

Fig. 16. Necking strain as a function of normalized imperfection wavelength. The plates are subjected to a constant overall stretching rate specified by $\dot{\lambda} h^{0} / \sqrt{\sigma_{R} / \rho}$. The material has hardening exponent, $N=0.1$, with $\sigma_{R} / E=0.002$. Material strain-rate dependence is not incorporated. For a steel plate $\left(\rho=8000 \mathrm{~kg} / \mathrm{m}^{3}\right)$ with initial thickness, $h^{0}=0.02 \mathrm{~m}$, and $\sigma_{R}=400 \mathrm{MPa}$, the overall stretch-rates above correspond to $\dot{\lambda}=2000 \mathrm{~s}^{-1}, 1000 \mathrm{~s}^{-1}, 500 \mathrm{~s}^{-1}, 200 \mathrm{~s}^{-1}$ and $100 \mathrm{~s}^{-1}$. 


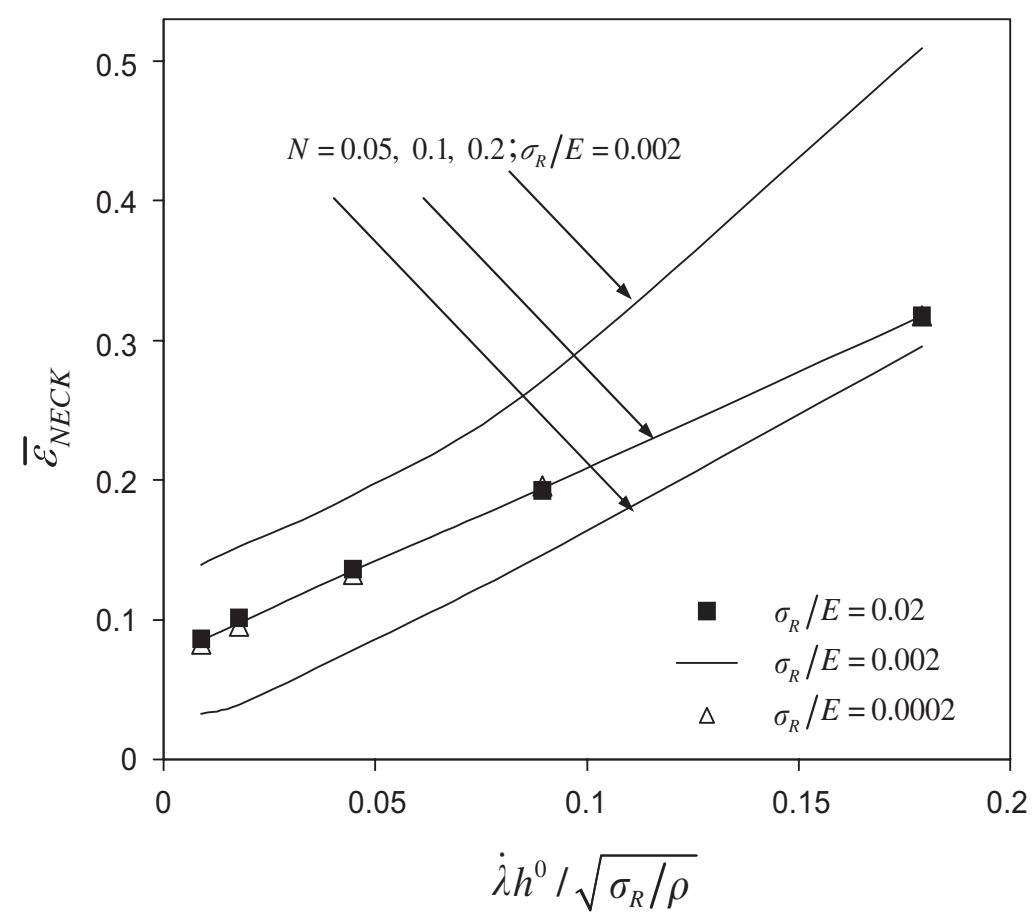

Fig. 17. Minimum necking strain as a function of normalized stretch-rate for several hardening exponents, $N$. The imperfection wavelength ratio associated with the minimum necking strain is $L^{0} / h^{0} \cong 3$ over the parameter range plotted. The imperfection amplitude is $\eta_{0}=0.04$. Material strain-rate dependence is not incorporated.

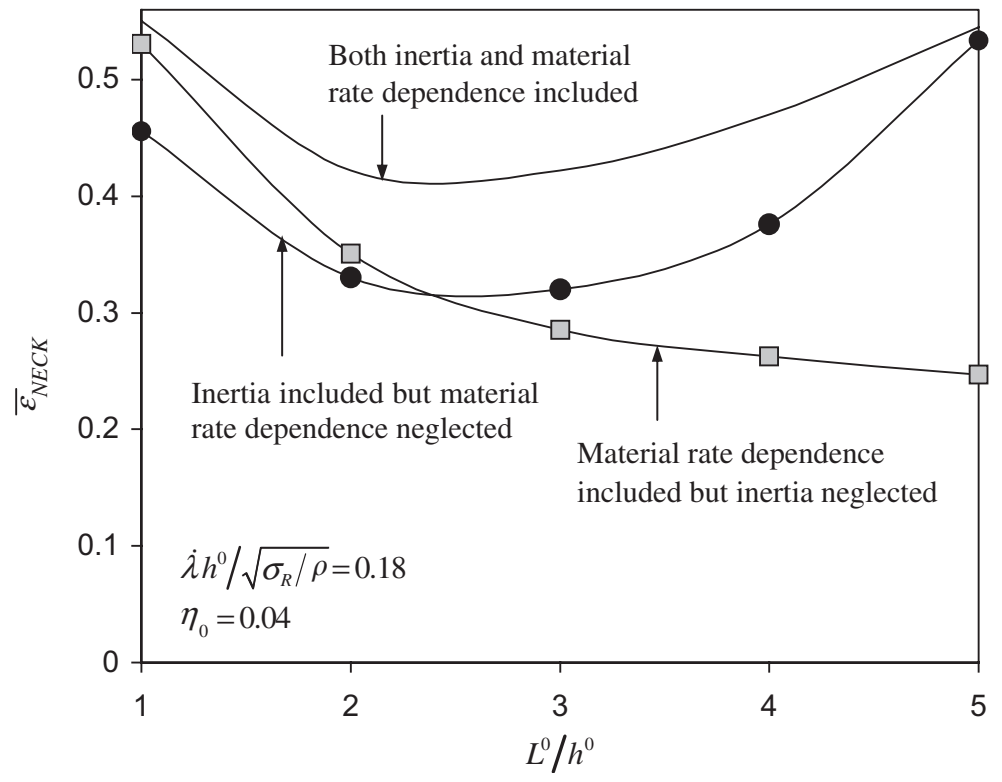

Fig. 18. Necking strains illustrating the roles of inertia and material rate-dependence acting independently and together. The material is specified by the overstress stress-strain relation (14) with $N=0.1, \sigma_{R} / E=0.002$ and $p=5$. If material rate-dependence is neglected, $\dot{\varepsilon}_{R} \rightarrow \infty$; if it is taken into account, $\dot{\varepsilon}_{R} h^{0} / \sqrt{\sigma_{R} / \rho}=0.36$. The rate-dependent material properties are representative of 304 stainless steel. For a plate with $h^{0}=0.02 \mathrm{~m}, \dot{\lambda}=2000 \mathrm{~s}^{-1}$ and $\dot{\varepsilon}_{R}=4000 \mathrm{~s}^{-1}$.

$\dot{\lambda} h^{0} / \sqrt{\sigma_{R} / \rho}$, for several values of the dimensionless material rate parameter, $\dot{\varepsilon}_{R} h^{0} / \sqrt{\sigma_{R} / \rho}$, including the rateindependent with $\dot{\varepsilon}_{R} \rightarrow \infty$. At low stretch rates it is again seen that material rate effects are small due to the character of the overstress relation, as discussed earlier. However, at higher stretch rates, when $\dot{\lambda}$ becomes comparable with $\dot{\varepsilon}_{R}$, material rate effects contribute significantly to neck retardation. As previously 


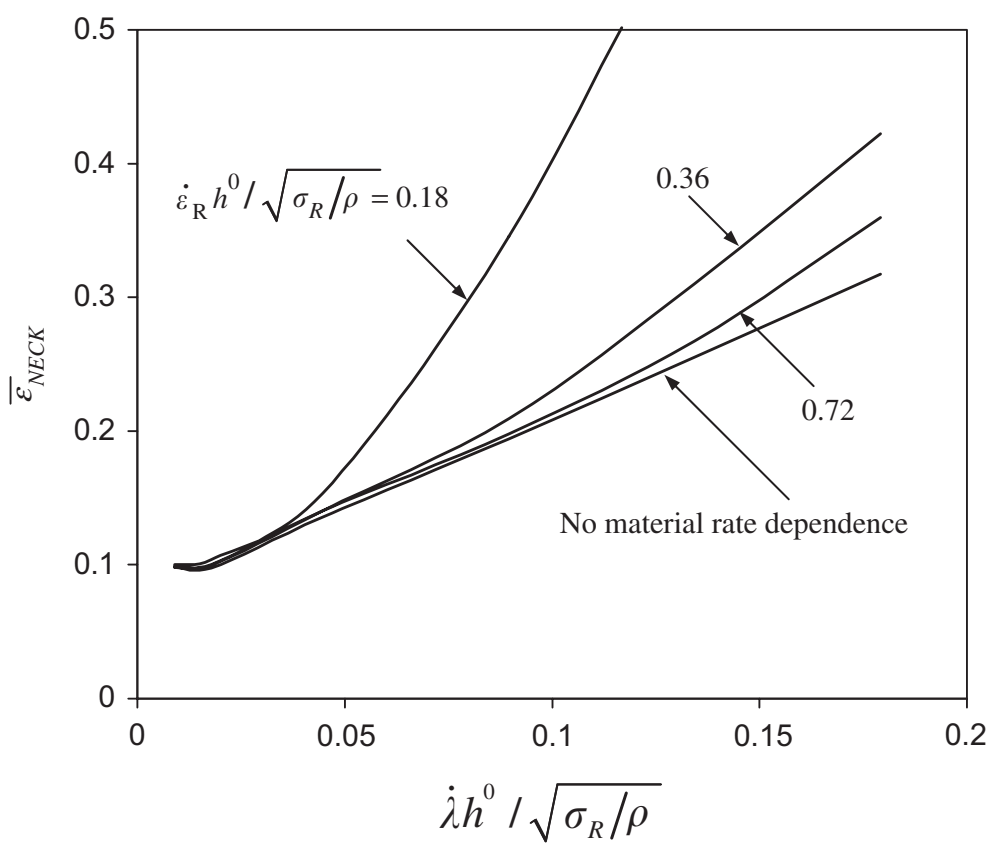

Fig. 19. Near-minimum necking strain (associated with $L^{0} / h^{0}=3$ ) as a function of dimensionless stretch-rate for material characterized by overstress relation (14) with $N=0.1, \sigma_{R} / E=0.002, p=5$ and $\eta_{0}=0.04$. The curve for no material rate-dependence has $\dot{\varepsilon}_{R} \rightarrow \infty$ in (14).

emphasized, the minimum of the necking strain with respect to the normalized imperfection wavelength, $L^{0} / h^{0}$, is very shallow implying a wide range of wavelengths are likely to be observed.

\section{Conclusions}

Necking behavior under dynamic stretching is highly nonlinear and is influenced by material behavior and inertial effects in a complex manner. This paper has attempted to unravel this interdependence with particular emphasis on neck retardation. In doing so, use has been made of a combination of results, some based on perturbation methods, which are valid in the very early stages of neck growth, and others based on a simple nonlinear one-dimensional, two-state model. The two-state model captures most of the parametric dependencies except for the dependence of the necking strain on imperfection wavelength. It has been supplemented by extensive finite element calculations of periodic neck development in plates having initial sinusoidal imperfections. A brief summary of the main findings follow.

(i) The essential dimensionless stretch-rate characterizing the role of inertia on necking is $\dot{\lambda} h^{0} / \sqrt{\sigma_{R} / \rho}$. Multiple necks become a dominant feature when $\dot{\lambda} h^{0} / \sqrt{\sigma_{R} / \rho} \sim 0.01$ and significant neck retardation due to inertia occurs for larger values of this parameter.

(ii) The wavelength of the initial imperfection in the undeformed state that leads to the lowest necking strain was found to be $L^{0} / h^{0} \cong 3$ over the parameter range explored in the finite element calculations. However, there is a broad range of initial wavelengths that give nearly the same necking strain suggesting a strong competition for dominance when the imperfection is not purely sinusoidal in shape. When inertia is not important, long wavelength initial imperfections are the most critical and the necking strain is nearly independent of the normalized imperfection wavelength if $L^{0} / h^{0} \geqslant 3$. The Shenoy-Freund perturbation analysis of undulation growth discussed in Section 2.2 predicts the necks that grow the fastest in early stages will have somewhat larger values of $L / h$ than $L^{0} / h^{0} \cong 3$. In part, this difference can be accounted for by noting that $L / h$ in the Shenoy-Freund analysis represents the dimensionless wavelength in the deformed state when the overall strain is at the onset of neck growth whereas $L^{0} / h^{0}$ is defined in terms of quantities in undeformed state. Thus, for example, $L / h=1.49 L^{0} / h^{0}$ when $\bar{\varepsilon}=0.2$, 


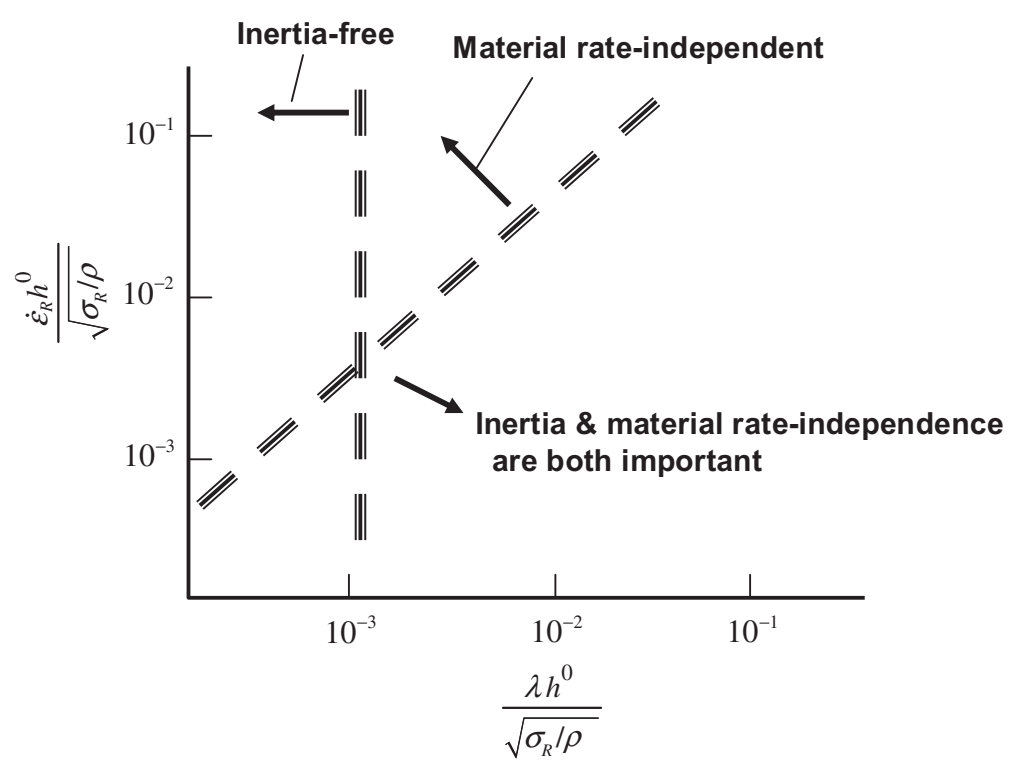

Fig. 20. Domain map for influence of inertia and material strain-rate dependence for a material characterized by the overstress relation (14).

and $L / h=1.82 L^{0} / h^{0}$ when $\bar{\varepsilon}=0.3$. It is also likely that necking growth rates change as the necks develop and behavior becomes highly nonlinear.

(iii) Imperfections must be invoked to analyze necking of an otherwise perfect specimen whenever either material rate effects or inertia are important. Bifurcation from a solution for a perfect configuration does not occur, unlike the situation for quasi-static straining of rate-independent materials. Moreover, the imperfection amplitude can have a significant influence on the necking strain, as illustrated by the twostate results in Fig. 13. The dependence on the amplitude of the imperfection has been calculated using the finite element method for the case of sinusoidal imperfection shapes and found to be comparable to those in Fig. 13. In the present study, the material is assumed to have unlimited ductility. Damage in the form of void growth and coalescence has been taken into account in studies of necking and fragmentation in dynamic ring expansion by several authors, including Pandolfi et al. (1999), Sorensen and Freund (2000) and Becker (2002). Since inertia and material rate-dependence increase the necking strain, it is likely that material damage will emerge at high rates of stretch possibly even before significant necks develop. It should be noted, however, that material rate-dependence and inertia will still contribute beneficially to neck retardation even if damage is occurring. Needleman (1991) observed that stress triaxiality is reduced in a neck forming dynamically relative to that under quasi-static conditions, and this is beneficial when the damage mechanism is void growth.

(iv) The interplay between material rate-dependence and inertia in neck retardation is complex and dependent on the precise form of the constitutive model. It was noted (cf. Fig. 12) that a power-law dependence on the strain-rate (1) leads to neck retardation over the entire range of stretch-rates, including the quasi-static limit. By contrast, rate effects in the overstress constitutive relation (14) only come into play at higher strain-rates and have no influence on the quasi-static limit. It is important that material strain-rate hardening be correctly characterized. For the overstress relation (14), the parameter map in Fig. 20 shows the regimes dominated by either material rate-dependence or inertia, and it also reveals the regime in which both should be taken into account.

\section{Acknowledgment}

This work was supported in part by ONR Grant N00014-04-1-0154 and in part by the School of Engineering and Applied Sciences, Harvard University. 


\section{Appendix A. Growth rate of small undulations under dynamic stretching}

Here the equation governing the growth of small undulations under dynamic plane strain stretching as given by Shenoy and Freund (1999) is translated into a form appropriate to sinusoidal undulations on an infinitely long plate. The reader is referred to the Shenoy-Freund paper for full details not covered here. The analysis assumes a constant overall stretch rate, $\dot{\lambda}$. The notation and dimensionless variables introduced in Section 1.1 are employed. Coordinates $\left(x_{1}, x_{2}\right)$ are aligned with and perpendicular to, respectively, the mid-plane of the plate. The perturbation in the velocity component in the $x_{2}$-direction relevant to the range of parameters presented in this paper has the form

$$
v_{2}=\sin \left(\frac{2 \pi x_{1}}{L}\right)\left\{A \Re\left[\sin \left(\frac{p x_{2}}{h}\right)\right]+B \mathfrak{I}\left[\sin \left(\frac{p x_{2}}{h}\right)\right]\right\}\left\{C \mathrm{e}^{\mathrm{t} / t^{*}}+D \mathrm{e}^{-\mathrm{t} / t^{*}}\right\}
$$

where $\Re()$ and $\mathfrak{I}($ ) denote real and imaginary parts, $A, B, C, D$ are real constants, and $p$ is any root of

$$
\begin{aligned}
& {\left[\frac{1}{4 N}-\frac{1}{2} \frac{\sigma}{E_{\mathrm{t}}}\right] p^{4}+\left[\left(1-\frac{1}{2 N}\right)\left(\frac{2 \pi h}{L}\right)^{2}+\left(\frac{\dot{\lambda} h / \sqrt{E_{\mathrm{t}} / \rho}}{\dot{\lambda} t^{*}}\right)^{2}\right] p^{2}} \\
& +\left(\frac{2 \pi h}{L}\right)^{2}\left[\left(\frac{1}{4 N}+\frac{1}{2} \frac{\sigma}{E_{\mathrm{t}}}\right)\left(\frac{2 \pi h}{L}\right)^{2}-\left(2\left(\frac{\dot{\lambda} h}{\sqrt{E_{\mathrm{t}} / \rho}}\right)^{2}-\left(\frac{\dot{\lambda} h / \sqrt{E_{\mathrm{t}} / \rho}}{\dot{\lambda} t^{*}}\right)^{2}\right)\right]=0 .
\end{aligned}
$$

Shenoy and Freund identified four regimes, depending on the complex character of the roots $p$ of (A.2). Over the range of parameters presented in this paper, $p$ is a complex number with nonzero real and imaginary parts such that (A.1) is the relevant representation. For the infinite plate, $\dot{\lambda} h / \sqrt{E_{\mathrm{t}} / \rho}$, emerges as the dimensionless stretch-rate as evident in (A.2). We have followed Shenoy and Freund by taking $\dot{\lambda} t^{*}$ as the dimensionless time scale characterizing the undulation growth rate. However, by (A.2), it can be noted that an alternative choice for the second parameter could be $t^{*} \sqrt{E_{\mathrm{t}} / \rho} / h$. The homogeneous boundary conditions are

$$
\begin{aligned}
& A \Re\left[\left(\left(\frac{2 \pi h}{L}\right)^{2}-p^{2}\right) \sin \left(\frac{p}{2}\right)\right]+B \mathfrak{I}\left[\left(\left(\frac{2 \pi h}{L}\right)^{2}-p^{2}\right) \sin \left(\frac{p}{2}\right)\right]=0, \\
& A\left\{\Re \left[\left(\left(\frac{1}{4 N}-\frac{1}{2} \frac{\sigma}{E_{\mathrm{t}}}\right) p^{3}+\left(\left(1-\frac{1}{4 N}-\frac{1}{2} \frac{\sigma}{E_{\mathrm{t}}}\right)\left(\frac{2 \pi h}{L}\right)^{2}+\left(\frac{\dot{\lambda} h / \sqrt{E_{\mathrm{t}} / \rho}}{\dot{\lambda} t^{*}}\right)^{2}\right) p\right)\right.\right. \\
& \left.\left.\quad \times \cos \left(\frac{p}{2}\right)+\left(\frac{\dot{\lambda} h}{\sqrt{E_{\mathrm{t}} / \rho}}\right)^{2}\left(\frac{2 \pi h}{L}\right)^{2} \sin \left(\frac{p}{2}\right)\right]\right\} \\
& \quad+B\left\{\mathfrak{I}\left[\left(\frac{1}{4 N}-\frac{1}{2} \frac{\sigma}{E_{\mathrm{t}}}\right) p^{3}+\left(\left(1-\frac{1}{4 N}-\frac{1}{2} \frac{\sigma}{E_{\mathrm{t}}}\right)\left(\frac{2 \pi h}{L}\right)^{2}+\left(\frac{\dot{\lambda} h / \sqrt{E_{\mathrm{t}} / \rho}}{\dot{\lambda} t^{*}}\right)^{2}\right) p\right)\right. \\
& \left.\left.\quad \times \cos \left(\frac{p}{2}\right)+\left(\frac{\dot{\lambda} h}{\sqrt{E_{\mathrm{t}} / \rho}}\right)^{2}\left(\frac{2 \pi h}{L}\right)^{2} \sin \left(\frac{p}{2}\right)\right]\right\}=0 .
\end{aligned}
$$

Solutions require the determinant of this system to vanish.

\section{References}

ABAQUS, 2004. ABAQUS/Explicit User's Manual Version 6.5, ABAQUS Inc.

Becker, R., 2002. Ring fragmentation predictions using the Gurson model with material stability conditions as failure criterion. Int. J. Solids Struct. 39, 3555-3580.

Fressengeas, C., Molinari, A., 1994. Fragmentation of rapidly stretching sheets. Eur. J. Mech. A Solids 2, 251-268.

Grady, D.E., Benson, D.A., 1983. Fragmentation of metal rings by electromagnetic loading. Exp. Mech. 12, 393-400. 
Guduru, P.R., Freund, L.B., 2002. The dynamic of multiple neck formation and fragmentation in high rate extension of ductile materials. Int. J. Solids Struct. 39, 5615-5632.

Han, J.-B., Tvergaard, V., 1995. Effect of inertia on the necking behavior of ring specimens under rapid radial expansion. Eur. J. Mech. A Solids 14, 287-307.

Hill, R., Hutchinson, J.W., 1975. Bifurcation phenomena in the plane strain tension test. J. Mech. Phys. Solids 23, $239-264$.

Hutchinson, J.W., Neale, K.W., 1977. Influence of strain-rate sensitivity on necking under uniaxial tension. Acta Metall 25, 839-846.

Hutchinson, J.W., Neale, K.W., Needleman, A., 1978. Sheet necking-I. Validity of plane stress assumptions of the long wavelength approximation. In: Koistinen, D.P., Wang, N.-W. (Eds.), Mechanics of Sheet Metal Forming. Plenum Publishing Corp., New York, NY, pp. 111-126.

Marciniak, Z., Kuczynski, K., 1967. Limit strains in the processes of stretch-forming sheet metal. Int. J. Mech. Sci. 9, 609-620.

Mercier, S., Molinari, A., 2003. Predictions of bifurcation and instability during dynamic extension. Int. J. Solids Struct. 40, $1995-2016$.

Needleman, A., 1991. The effect of material inertia on neck development. In: Yang, W.H. (Ed.), Topics in Plasticity. AM Press, Ann Arbor, MI, pp. 151-160.

Niordson, F.I., 1965. A unit for testing materials at high strain rates. Exp. Mech. 5, 29-32.

Pandolfi, A., Krysl, P., Ortiz, M., 1999. Finite element simulation of ring expansion and fragmentation: the capturing of length and time scales through cohesive models of fracture. Int. J. Fract. 95, 279-297.

Regazzoni, G., Johnson, J.N., Follansbee, P.S., 1986. Theoretical study of the dynamic tensile test. J. Appl. Mech. 53, 519-528.

Shenoy, V.B., Freund, L.B., 1999. Necking bifurcations during high strain rate extension. J. Mech. Phys. Solids 47, $2209-2233$.

Sorensen, N.J., Freund, L.B., 2000. Unstable neck formation in a ductile ring subjected to impulsive radial loading. Int. J. Solids Struct. $37,2265-2283$.

Storen, S., Rice, J.R., 1975. Localized necking in thin sheets. J. Mech. Phys. Solids 23, 421-441.

Tuggu, P., Neale, K.W., Lahoud, A.E., 1990. Inertial effects on necking in tension. Int. J. Solids Struct. 26, $1275-1285$.

Woodford, D.A., 1969. Strain-rate sensitivity as a measure of ductility (strain-rate sensitivity measurements to determine ductility of materials, noting relation to elongation). ASM. Trans. Q. 62, 291-293.

Zhang, H., Ravi-Chandar, K., 2006. On the dynamics of necking and fragmentation: I. Real-time and post-mortem observations in Al 6061-O. Int. J. Fract. 142, 183-217. 